\title{
Dynamic Competition with Network Externalities: How History Matters*
}

\author{
Hanna Halaburda \\ Bank of Canada ${ }^{\dagger} \&$ NYU-Stern \\ Bruno Jullien \\ Toulouse School of Economics, CNRS, Toulouse \\ Yaron Yehezkel \\ Coller School of Management \\ Tel-Aviv University
}

September 2013, revised July 26, 2019

\footnotetext{
*For helpful comments and discussion we thank Gary Biglaiser, Tim Bresnahan, Luis Cabral, Carlo Cambini, Annamaria Conti, Jacques Crémer, Renato Gomes, Andrei Hagiu, Pedro Pereira, Markus Reisinger, and Andre Veiga as well as participants at the Fourth Annual Searle Center Conference on Internet Search and Innovation, the 11th Workshop on Media Economics, the Third ICT Conference in Munich, the Multi-Sided Platforms Workshop at NUS, the Conference on the Economics of ICT in Paris, the ZEW Conference on the Economics of ICT in Mannheim, the 8th Taller in Industrial Organization, the 2015 Conference on Economics of Intellectual Property, Software and the Internet in Toulouse, the NYU Stern Economics of Strategy Workshop, the CRES Strategy Conference in St. Louis and the ESMT Workshop on the Economics of Platforms in Berlin. We gratefully acknowledge financial support from the NET Institute, www.NETinst.org, the Henry Crown Institute of Business Research in Israel and ANR under grant ANR-17-EURE-0010. This project has also received funding from the European Research Council (ERC) under the European Union's Horizon 2020 research and innovation programme (grant agreement No 670494).

${ }^{\dagger}$ The views expressed in this paper are those of the authors. No responsibility for them should be attributed to the Bank of Canada.
} 


\begin{abstract}
We consider dynamic competition among platforms in a market with network externalities. A platform that dominated the market in the previous period becomes "focal" in the current period, in that agents play the equilibrium in which they join the focal platform whenever such equilibrium exists. Yet when faced with higher-quality competition, can a low-quality platform remain focal? In the finite-horizon case, the unique equilibrium is efficient for "patient" platforms; with an infinite time horizon, however, there are multiple equilibria where either the low- or high-quality platform dominates. If qualities are stochastic, the platform with a better average quality wins with a higher probability, even when its realized quality is lower, and this probability increases as platforms become more patient. Hence social welfare may decline as platforms become more forward looking.
\end{abstract}

JEL Classification : L1

Keywords: network externalities, dynamic competition, coordination

\title{
1 Introduction
}

A traditional concern in markets with network externalities is that the "wrong" platform may dominate due to consumers' miscoordination. Our main research question is whether markets can correct such inefficient outcomes in a dynamic competition between far-sighted platforms, when consumers' expectations concerning the dominant platform depend on the history. We confirm this intuition for long finite horizon, but identify two novel factors that may prevent efficient outcome when the horizon is infinite.

Platform competition typically involves both network effects and repeated interaction. We often observe that a platform that was dominant in the recent past has the advantage of customers' favorable expectations, meaning that customers expect that this platform will also attract other customers in the current period. We shall refer to such a platform as a focal platform. For example, Apple's success with the iPhone 4 resulted in pre-orders for its iPhone 5 exceeding 2 million within a day of its launch in September 2012 - even though there were not yet any applications that could take advantage of the phone's new features. Moreover, analysts predicted that 50 million users would buy the new smartphone within three months of its launch. ? $^{1}$ A similar dynamics was in evidence for the iPhone 6's release, as sales topped 4 million in the first

\footnotetext{
${ }^{1}$ Ryan Faughnder and Adam Satariano, "Apple iPhone 5 Pre-Orders Top 2 Million, Doubling
} 
24 hours..$^{2}$ Analysts reported that this trend continued with the iPhone 7's release.$^{3}$ In contrast, neither Blackberry nor Windows phones enjoyed a comparable advantage during this period. Even though the Blackberry phones - the Q10 and the Z10 received glowing reviews, the absence of positive expectations made it difficult for the firm to gain substantial market share: application developers were skeptical about the phone's ability to attract users; sales were indeed sluggish, which was due in no small part to the paucity of available apps 4 We can ascribe the developer' skepticism and the resulting lack of apps to the Blackberry platform's recent history.

Yet even dominant platforms can lose market share, despite winning in the past, when faced with a higher-quality competitor. In the market for smartphones, for instance, Nokia dominated the early stage (along with RIM) with smartphones based on a physical keyboard. Apple then revolutionized the industry by betting on its new operating system, which featured touch-screen technology. A few years later, Samsung managed to gain substantial market share (though not market dominance) by betting on smartphones with large screens. The supplanting of industry leaders was likewise a common theme in the market for video-game consoles. Nintendo, Sony, and Microsoft alternated as the market leader (Hagiu and Halaburda 2009). Thus platforms are sometimes able to overcome the market's unfavorable expectations. These examples raise the question of when is it profitable for a platform facing unfavorable position to invest in capturing the market, and when it is profitable for a platform facing a favorable position to invest in retaining the market, if each firm knows that its current strategy may affect its future position.

If the impact on the future is ignored, then it may not be profitable for the nonfocal platform to overcome unfavorable expectations even if it can offer higher quality than the focal platform. The reason is that network effects provide the focal platform with a short-term competitive advantage: in a one-time interaction, a focal platform can use its position to dominate the market even when competing against a higher-quality platform. Yet we can expect that, in the long run, if platforms are forward looking then a high-quality but presently nonfocal platform can overcome its expectations disadvantage because it can afford short-term losses in order to become focal in the future. At the same time, a low-quality forward looking focal platform also has an incentive to invest in maintaining its dominant market position. So even though the

Record", Bloomberg (2012). Available at: (http://www.bloomberg.com/news/2012-09- 17/appleiphone-5-pre-orders-top-2-million-double-prior-record-1-.html).

${ }^{2} \mathrm{http}: / /$ www.cnet.com/news/apple-iphone-6-iphone-6-plus-preorders-top-4m-in-first-24-hours

${ }^{3}$ https://www.ft.com/content/dc40349c-8d2b-345c-b9c3-20dfe41a9992

${ }^{4}$ On the quality and launch of the Blackberry phones, see Austen (2012) and Bunton (2013). 
nonfocal platform has a quality advantage, the focal platform has an expectations advantage.

In this paper, we ask whether a low-quality platform currently benefiting from its focal position can continue to dominate the market in a dynamic setting - that is, when platforms account for the future benefits of capturing the market today. For example, would Apple continue to dominate the market for tablets if competitors (Samsung's Tab, Microsoft's Surface, etc.) offered tablets of higher base quality? Is it possible for a video-game console to maintain market leadership when facing higherquality competitors? More specifically, we are interested in whether the higher-quality platform's likelihood of winning increases with the importance attached by firms to the future. The winning platform's identity affects not only the firms involved but also social welfare, which is higher when the better platform wins.

To investigate this research question, we analyze a model of dynamic competition between two platforms. In each period, one of the platforms wins by capturing all of the market. So as to focus on the model's dynamic aspects, we assume that customers are homogeneous $5^{5}$ Consumers base their current-period behavior on their observation of past outcomes; thus the platform that won the market in the previous period becomes focal in the current period. It follows that capturing the market in one period gives the platform an advantage in future periods. Hence a nonfocal platform may be willing to sacrifice current profits to gain a better future market position.

We start with the case where each platform's stand-alone quality is constant for all periods and where the time horizon is finite. We show that, when platforms do not care about future profits, the low-quality focal platform maintains its position despite the nonfocal platform's higher quality — provided the quality gap is not too great. But when the future is important for the platforms or the quality gap is sufficiently large, the higher-quality platform wins the market at the start of the game and maintains its leadership. This outcome follows because a high-quality platform can earn higher profits than the low-quality one as the focal platform in the last period. So as compared with the low-quality platform, the high-quality platform has a larger incentive to fight for focality in the game's early stages.

We then consider the infinite-horizon case. We find that, when platforms care moderately about the future, there is a unique equilibrium in which a high-quality platform wins the market. But when the platforms care a lot about the future, the result is an increase both in the focal platform's incentive to maintain its position

\footnotetext{
${ }^{5}$ The consequences of this assumption are addressed in Section 7 .
} 
and in the nonfocal platform's incentive to win the focal position. This gives rise to multiple Markov equilibria. In one Markov equilibrium, the high-quality platform wins the focal position in the first period and then maintains it indefinitely - even if it begins as a nonfocal platform; however, there are equilibria in which the focal platform can maintain its leadership indefinitely even if it is of low quality. Thus an infinite horizon yields a new form of market failure, leading to an outcome where one platform aggressively builds market share under all circumstances (i.e., both on and off the equilibrium path) and succeeds in doing so because the other platform restrains from sacrificing current profit.

In terms of social welfare, these results indicate that when firms' patience increases from low to moderate, the social welfare increases. This outcome reflects the market's movement from the equilibrium in which the low-quality platform wins to the equilibrium in which the high-quality one overcomes its nonfocal position and thereafter maintains its newly acquired focal position indefinitely. However, as firms' patience increases further, the effects on welfare are ambiguous owing to the existence of multiple equilibria.

These results are a unique consequence of network effects. We consider an alternative model where instead of network effects buyers face switching costs and platforms can price discriminate between existing and new consumers. We find that the market failure that our paper identifies vanishes. Under switching costs, when players sufficiently care about future payoffs, there is a unique equilibrium in which the best platform wins. Intuitively, in the absence of network effects, a buyer's decision to switch from one platform to the other is not affected by the buyer's beliefs concerning the decisions of other buyers. This result highlights the qualitative difference between network effects and switching costs as well as the important role that beliefs play in markets with competing platforms. ${ }^{6}$

When qualities are constant, our model finds that the same platform dominates the market in all periods. Yet there are some cases in which platforms "take turns" at being the dominant platform, as with the Sony, Nintendo, and Microsoft video-game consoles mentioned previously. We therefore study how focality and the importance of the future affect changes in market leadership and market efficiency.

To study this question, we consider the case where the quality of each platform changes stochastically every period - a setup that is consistent with the continuous technology improvements seen in the markets for such products as video-game consoles

\footnotetext{
${ }^{6}$ For an extensive discussion of switching costs, see Farell and Klemperer (2007).
} 
and smartphones. In this setting it is possible for a low-quality platform to itself become a higher-quality one. Even so, we will assume that one of the platforms is of higher quality in average.

Unlike the case of fixed quality, in this stochastic scenario there is a unique Markov equilibrium. It is possible for either platform to win the market in any period if its quality in that period is sufficiently high. Still, the more platforms care about the future, the more likely it is that the platform with better average quality wins the market, even when its quality realization is lower. In some cases it is also possible for a focal platform to lose its market dominance even if its quality realization is higher than that of the nonfocal competitor. Thus social welfare may decline with increases in platforms' concern for the future.

The intuition behind this result is as follows. The platform with a higher quality on average is more likely capable of defending its focal position. Therefore, as future considerations become more important to platforms, this firm has more incentive to compete aggressively in order to capture a focal position even if its current realized quality is low. At the same time, the platform expecting a lower future quality on average has less incentive to win the market even if its current quality is high. This result indicates that the changes in market leadership, following technological improvements, which we observe in several markets for platforms (e.g., video-game consoles, smartphones), may not necessarily result in outcome in which the platform with higher quality wins.

\section{Related Literature}

Our paper's main conclusion is that even long-term considerations may not lead to an outcome in which the best platform wins. For example, there is disagreement in the economics literature as to whether the presence of network effects leads to long-term market inefficiency. David (1985) argues that the QWERTY keyboard's prevalence is an example of long-term inefficiency due to network effects; that claim is based on evidence that the Dvorak keyboard enables faster typing and requires less training. Liebowitz and Margolis (1990) criticize David's argument by claiming, on the basis of a case study, that the success of QWERTY is due not to network effects but rather to its superior quality vis-à-vis Dvorak. In an experiment, Hossain and Morgan (2009) find that the more efficient platform always wins over time, which would seem to support the claim of Liebowitz and Margolis. Our paper contributes to this debate by 
demonstrating that, when platforms strategically set prices to compete for users, both efficient and inefficient equilibria are possible in the long term.

Most theoretical analyses of platform competition focus on static games. Caillaud and Jullien $(2001,2003)$ introduce the notion of favorable beliefs - with respect to networks in the context of two-sided markets - as a tool that can be used to characterize the full equilibrium set for competition between undifferentiated platforms. This concept was used in subsequent research on two-sided markets (see Hagiu 2006; Jullien 2011; Halaburda and Yehezkel 2013) as a way of modeling market leadership when one platform benefits from favorable expectations; it has also been used in the literature on telecommunications to model consumer inertia (Lopez and Rey 2016). Though all of these papers acknowledge the dynamic nature of platform competition, their aim is to approximate market characteristics using static models. Halaburda and Yehezkel (2016) extend this concept to partial beliefs advantage and explore how platform's pricing strategies affect their future profits; the article employs a simple multi-period setup in which the extent of beliefs advantage depends on the market's history.

There has been some work addressing dynamic price competition between platforms. Our main contribution to this literature is showing how beliefs depending on the history of the market affect dynamic competition between vertically differentiated platforms. Doganoglu (2003), Cabral (2011) and Biglaiser and Crémer (2016) consider platforms that are identical in their intrinsic qualities, while we focus on the tradeoff between network effects and quality. Interestingly, they show that an entrant with a smaller network may be active in the market, despite lack of intrinsic quality advantage. This result is possible with sufficient customer heterogeneity and switching costs.7 Customer heterogeneity allows for niche firms to exists and periodically gain strong position, despite network effects.

Chen and Tse (2008) characterize the optimal dynamic trajectory of prices in platform competition. They focus on an environment where horizontal differentiation, heterogeneity of users and multi-homing allow for multiple platforms to coexist in the market in the equilibrium.

\footnotetext{
${ }^{7}$ Our paper abstracts from switching costs in order to focus on the dynamics of consumer expectations. Consequently, consumers in our model need not form beliefs about the market's future. The real-life examples that we cite (i.e., the markets for smartphones and video games) may include both network externalities and switching costs (stemming, for example, from the adjustments required after adoption of a new operating system). However, we share with those other models the dynamic-game feature of firms competing aggressively to build their market share in the current period so as to gain a long-term advantage. For an analysis of such strategies in games involving dynamic competition, see Besanko, Doraszelski, and Kryukov (2014).
} 
Mitchell and Skrzypacz (2006) analyze dynamic competition between platforms of different intrinsic qualities and show that network effects trumps quality, resulting in excess inertia, in a short run. But the quality wins in the long run. This is the property of the unique Markov equilibrium they obtain in the infinite game. Since they assume sufficient horizontal differentiation, for any prices there exists a unique allocation of customers attracted to each platform. Thus, they can abstract from the coordination problem. In contrast, we focus on environments where network effects are much stronger than differences in taste. In such environments, coordination problem arises every period and beliefs play important role in the equilibrium. We show that with beliefs depending on the history of the market multiple Markov equilibria exist. Most importantly, there exist equilibria with inefficient firm winning and holding the market even when platforms are patient.

Fudenberg and Tirole (2000) find almost opposite effect to Mitchell and Skrzypacz (2006). In Fudenberg and Tirole (2000) excess inertia occurs when platforms are patient. For inpatient platforms, higher quality wins. Like us, they analyze environment where network effects are more important than heterogeneity of preference, giving rise to coordination problem. Crucially, they assume that consumers coordinate on the platform that offers them the highest quality. Such beliefs do not depend on the history of the market, and thus do not play a role in the dynamics. Our paper is the first one to address the dynamics of consumer expectation and how it affects the equilibria.

Argenziano and Gilboa (2012) consider a repeated coordination game where players use the game's history to form beliefs regarding the behavior of other players. Our paper adopts that approach in the context of platform competition, as we study how platforms should compete given such belief formation by consumers. But we add the feature that each platform can alter beliefs by capturing the market and thereby shifting the coordination of consumers in its favor. In a recent paper, Akerlof, Holden and Rayo (2018) adopted a related approach to address the issue of dynamic coordination in a market with network effects. They focus on monopoly dynamic pricing while our paper focuses on dynamic competition.

The rest of the paper is organized as follows. After describing the model in Section 2, in Section 3 we consider the benchmark case of a dynamic game with finite horizon. Section 4 characterizes Markov equilibria under an infinite time horizon, and Section 5 discusses the distinction between network effects and switching costs. Section 6 considers the case where platform qualities change stochastically over time. We conclude in Section 7 by summarizing our results and touching on some related 
considerations 8

\section{The Model}

Consider a homogeneous consumer population of size 1 and two competing platforms, $i=A, B$, with the same cost (normalized to 0 ) $9^{9}$ There are $T$ periods, $t=1,2, \ldots, T$, where $T$ may be finite or infinite. Each platform $i$ offers to the customers a good or service generating stand-alone value, $q_{i}>0$, which we refer to as quality ${ }^{10}$ Additionally, consumers, benefit from network effects. A consumer's utility from buying the good or service of (thereafter joining) platform $i$ is $q_{i}+\beta n_{i}-p_{i}$; here $n_{i}$ is a measure of the other consumers who have joined $i, \beta$ denotes the strength of network effects, and $p_{i}$ is the price of platform $i$.

Notice that our model makes two simplifying assumptions. First, we focus on one-sided network effects. We view this assumption as a reduced form to scenarios with both direct and indirect network effects. We make this simplifying assumption because of our focus on incumbency advantage due to a focal position. Some markets involve two-sided pricing with indirect network effects, yet, the features of the incumbency advantage are qualitatively similar to the incumbency advantage in our model ${ }^{11}$ Our second simplifying assumption is that consumers are homogeneous. In equilibrium, consumers join the same platform while the competing platform is empty. This raises the question of why should the empty platform keep competing in future periods. Intuitively, with heterogeneous consumers, some consumers may view their subjective preferences toward one of the platforms as more important than network effects. Consequently, both platforms will gain a positive market share, by serving at least their most loyal consumers. At the same time, platforms will also compete for consumers that do not have strong preference towards a specific platform, or consumers that view network effects as more important than their subjective preferences. Since the focus of this paper is on network effects, we focus our attention on these homogeneous consumers. We discuss the case of consumers' heterogeneous preferences in the conclusion and show in the online appendix that our insights extend to this case

\footnotetext{
${ }^{8}$ Online appendix presents results we obtain when combining network effects and switching costs, as well as an analysis with heterogeneous consumers.

${ }^{9}$ In Section 7 we discuss how our results are affected when agents are instead heterogeneous.

${ }^{10}$ We consider the case where the $q_{i}$ are fixed over time (Sections 3 and 4 ) and also the case where qualities change from one period to the next (Section 6).

${ }^{11}$ In a two-sided market, the nonfocal platform may adopt a "divide-and-conquer" strategy. Yet, adopting this strategy places the nonfocal platform at a competitive disadvantage in a similar way as in our model (see Caillaud and Jullien, 2001 and 2003).
} 
provided that network effects are strong enough.

Every period, each platform $i$ sets a price $p_{i}(t)$, and then consumers decide which platform to join for the current period. In what follows, a negative price is interpreted as one below cost 12 The two platforms operate for $T$ periods and discount future profits by $\delta$, where $0 \leq \delta<1$. There are no switching costs, so consumers' current payoff is not directly affected by past or future periods. Path dependency will arise solely from belief formation.

Competition in an environment with network effects often results in multiple equilibria, and it is also the case here. Consider the allocation of consumers that emerges for given prices. If $q_{i}-p_{i}(t)>q_{j}-p_{j}(t)+\beta$, then there is a unique consumers' allocation in which all consumers join platform $i$. Yet if

$$
\left|q_{A}-q_{B}+p_{B}(t)-p_{A}(t)\right|<\beta
$$

then there are two possible allocations: either all consumers join $A$ or all join $B$. This multiplicity makes it difficult to discuss dynamic competition in environments with network effects, and several solutions have been proposed to address the issue. We rely on the notion of pessimistic beliefs and a focal platform, as developed in Caillaud and Jullien (2003), Hagiu (2006), and Jullien (2011). When prices in period $t$ are compatible with multiple allocations of consumers, we say that platform $i$ is focal in period $t$ if the consumers' allocation is such that each consumer buys from platform $i$ if it is not a strictly dominant strategy to buy from platform $j$ and she is not indifferent between the two platforms ${ }^{13}$ As a consequence, under condition (1), all consumers join platform $i$. But a nonfocal platform $j$ can win the market if it sets $p_{j}$ low enough such that $q_{j}-p_{j}>q_{i}+\beta-p_{i}$. By contrast the focal platform $i$ can guarantee itself to win by setting $p_{i}$ such that $q_{j}-p_{j}<q_{i}+\beta-p_{i}$. We assume that in any period there is a focal platform.

In a dynamic model with $t=1, \ldots, T$, the identity of the focal platform in $t>1$ may be related to the market's history. In this paper we explore how allowing for such historical dependency affects the market's future outcomes, focusing on one-period dynamics.

During each period $t$, the market outcome is expressed by a pair $\left(w_{t}, f_{t}\right)$, where

\footnotetext{
${ }^{12}$ To allow for the possibility of negative prices, we must assume that agents who collect the resulting subsidy do indeed join the platform to the benefit of other users.

${ }^{13}$ This second condition aims at ensuring existence of equilibrium by allowing the nonfocal platform to sell in case of indifference. Indeed in all equilibria, prices will be such that consumers are indifferent and their action is made in such a way that it supports the equilibrium.
} 
$w_{t} \in\{A, B\}$ is the identity of the active platform - i.e., the platform that wins the market in $t^{14}$ - and $f_{t} \in\{A, B\}$ is the identity of the focal platform in $t$. Note that it is possible for the nonfocal platform to win the market. Based on their observation of past outcomes, consumers form conjectures about the platform most likely to win in the current period. These conjectures are assumed to converge to a single focal platform. In $t=0$, one of the platforms is arbitrarily set as the focal platform, which we call platform $A$. The identity of the focal platform $f_{t}$ is always common knowledge and is the only payoff-relevant variable in the next period. The dynamics of platform focality is then obtained by assuming that in any period, the last period's market winner becomes focal.

As a benchmark case for our analysis, consider a static one-period game in this environment. Network externalities may create market inefficiencies in the equilibrium of a static game. Although $A$ is the focal platform, it can be of higher or lower quality than platform $B$. In an equilibrium in which a focal platform $A$ wins, the losing platform $B$ sets the lowest price possible, $p_{B}=0$. Platform $A$ sets $q_{A}-p_{A}+\beta=q_{B}-0$ and earns $p_{A}=q_{A}-q_{B}+\beta$. Hence, such an equilibrium exists if $q_{A}-q_{B}+\beta>0$. Likewise, in an equilibrium in which a nonfocal platform $B$ wins, platform $A$ sets $p_{A}=0$ and platform $B$ sets $q_{B}-p_{B}=q_{A}-0+\beta$. Hence, such an equilibrium exists if $q_{A}-q_{B}+\beta<0$. Thus, if $q_{A}<q_{B}$ yet $q_{A}>q_{B}-\beta$, then platform $A$ wins despite offering lower quality. It wins because it happens to be focal. This effect is called excess inertia, and it leads to inefficient outcomes in equilibrium.

When there are multiple periods, a nonfocal platform may find it worthwhile to win the market by setting negative price in an earlier period. Doing so would yield the platform a negative current profit, but if the focal position is thereby captured then those losses could be recovered in future periods. Hence, in a dynamic market one could suppose that a higher-quality nonfocal platform is advantaged; after all, should it become focal, its higher quality yields greater profit than can be generated by a lower-quality platform. Hence the higher-quality platform has more incentive to invest in capturing the market than the lower-quality platform has to invest in defending its position. The focal platform anticipates this and strives to prevent the nonfocal platform from capturing the market.

\footnotetext{
${ }^{14}$ Market sharing allocations — where some consumers join platform $A$ while others join $B$ are unstable. In such allocations, all consumers are indifferent between joining platforms $A$ and $B$. Therefore, any deviation by a marginal consumer will tip the market to a stable allocation in which all consumers join the same platform. We therefore focus on allocation in which one of the platform dominates the market.
} 


\section{Dynamic game with finite horizon}

In this section we consider the case of a finite time horizon and show that there exists a unique subgame perfect Nash equilibrium. In this equilibrium, a high-quality but nonfocal platform wins the market in the first period and maintains its acquired focal position in all subsequent periods - provided that platforms care sufficiently about the future (i.e., $\delta$ is high and the time horizon is long) or that the quality gap between platforms $A$ and $B$ is sufficiently large. Otherwise, a low-quality platform that is focal in the first period will maintain that position thereafter.

Suppose that the time horizon is $T=2$. Our analysis will focus on the interesting case, where network effects are sufficiently large with respect to the quality gap, $\beta>$ $\left|q_{i}-q_{j}\right|$, so that the focal platform wins a one-period game even with a lower quality than the nonfocal platform.

Consider the last period, $t=2$. Since in this period there is no future, the subgame equilibrium is identical to the one-period benchmark described in Section 2. When platform $i$ is focal, it wins the market regardless of the quality gap; in this case, $i$ earns $q_{i}-q_{j}+\beta$ while the nonfocal platform $j$ earns 0 . Observe that the focal platform's lastperiod profits are greater when it is the higher-quality platform; formally, $q_{i}-q_{j}+\beta>$ $q_{j}-q_{i}+\beta$ when $q_{i}>q_{j}$.

We next turn to the first period, $t=1$. Recall that we assume that platform $A$ is focal in the first period. Each platform takes into account that capturing the market in this period will render it focal in the next period and earn it an additional profit of $q_{i}-$ $q_{j}+\beta$. An individual buyer, however, cannot affect the identity of the focal platform, as it depends on the buyer's beliefs concerning the behavior of other consumers.

Consider first an equilibrium in which $A$ wins the market in the first period. The lowest price that the losing platform $B$ is willing to charge in period 1 is $p_{B}=-\delta\left(q_{B}-\right.$ $\left.q_{A}+\beta\right)$. In order to convince buyers to join it, platform $A$ must charge a price such that $q_{A}+\beta-p_{A}=q_{B}-p_{B}$. Notice that the buyer's expected payoff in period 2 does not affect the buyer's decision at time 1 , because future payoffs depend only on future network effects, i.e., on the buyer's beliefs concerning the decisions of other buyers, and therefore do not depend on the buyer's choice of platform $A$ or $B$ in period 1 .

Setting $p_{A}=q_{A}+\beta-q_{B}+p_{B}$ in period 1 is the best reply of platform $A$ only if its profit is nonnegative. We thus have the condition

$$
q_{A}-q_{B}+\beta-\delta\left(q_{B}-q_{A}+\beta\right)+\delta\left(q_{A}-q_{B}+\beta\right) \geq 0 .
$$


The same analysis applies to an equilibrium in which the nonfocal platform $B$ wins in the first period. In this equilibrium, the losing platform $A$ charges a price $p_{A}=-\delta\left(q_{A}-q_{B}+\beta\right)$ and the winning platform $B$ must earn a positive profit at the best-reply winning price $p_{B}=q_{B}-\beta-q_{A}+p_{A}$. So now we have the following condition:

$$
q_{B}-q_{A}-\beta-\delta\left(q_{A}-q_{B}+\beta\right)+\delta\left(q_{B}-q_{A}+\beta\right) \geq 0 .
$$

Comparing conditions (2) and (3) reveals that for any set of parameters there exists exactly one equilibrium (except for the degenerate case where profit is zero for both platforms). In this unique equilibrium, a focal platform $A$ wins in both periods if

$$
q_{B}-q_{A}<\frac{\beta}{1+2 \delta}
$$

Platform $B$ wins in both periods otherwise. Hence, we conclude that the equilibrium is unique and has the following characteristics for a horizon $T=2$.

(i) If the quality differential is smaller than $\beta /(1+2 \delta)$, then the focal platform (platform $A$ ) wins the market in any subgame. In this case, the focal platform earns two-period profit of $(1+2 \delta)\left(q_{A}-q_{B}\right)+\beta$, which is higher than its profit in a one-period game, $q_{A}-q_{B}+\beta$, if and only if it is of higher quality.

(ii) If the quality differential lies between $\beta /(1+2 \delta)$ and $\beta$, then the focal platform wins in a one-period game while the higher-quality platform wins in both periods of a two-period game.

Thus a nonfocal platform $B$ with superior quality can win the market in the twoperiod case when it could not in the one-period case. The intuition for this result is that the higher-quality platform has more to win in becoming the last period focal platform. Since platforms care about the future and since $B$ earns more profit (than $A$ ) from being focal in the last period, it follows that platform $B$ 's incentive to capture the focal position in the first period is stronger than platform $A$ 's incentive to maintain its focal position. In particular, a high-quality but nonfocal platform can win the market in the first period and maintain its focal position in all periods, when $\delta$ is high enough and if the quality gap $\left(q_{B}-q_{A}\right)$ is sufficiently large. In such cases, forwardlooking platforms eliminate the inefficiency that might otherwise emerge because of the consumer coordination problem.

For longer horizon we can apply the same logic to solve the game backward and extend the conclusions to any horizon. The following lemma characterizes how, for 
an arbitrary finite horizon, the equilibrium outcome depends on the parameters (all proofs are in the appendix).

Lemma 1 (Subgame perfect equilibrium for arbitrary finite $T$ ) For any set of parameters $q_{A}, q_{B}, \beta>\left|q_{A}-q_{B}\right|$, and $\delta<1$, there exists a unique subgame perfect equilibrium for arbitrary finite $T$. In the equilibrium outcome, the same platform wins the market in all periods. The winning platform's identity and its future discounted profit depend on the parameters as follows.

(i) If $\left|q_{A}-q_{B}\right|<\beta(1-2 \delta) /\left(1-(2 \delta)^{T}\right)$, then the focal platform wins in any subgame and thus platform $A$ being focal in the first period, it wins the market and maintains its position with a total profit of

$$
\left(q_{A}-q_{B}\right) \frac{1-(2 \delta)^{T}}{1-2 \delta}+\beta
$$

(ii) If $\left|q_{A}-q_{B}\right|>\beta(1-2 \delta) /\left(1-(2 \delta)^{T}\right)$, then there exists an horizon $\hat{T}<T$, such that the focal platform wins the market in any subgame of horizon less or equal than $\hat{T}$, while the higher-quality platform wins the market in any subgame with horizon larger than $\hat{T}$. Thus platform A wins (in all periods) if it has superior quality, and then it earns

$$
\left(q_{A}-q_{B}+\beta\right) \frac{1-\delta^{T-\hat{T}}}{1-\delta}+\delta^{T-\hat{T}}\left(\left(q_{A}-q_{B}\right) \frac{1-(2 \delta)^{\hat{T}}}{1-2 \delta}+\beta\right)
$$

while platform B wins (in all periods) if it has superior quality, and then it earns

$$
\left(q_{B}-q_{A}+\beta\right) \frac{1-\delta^{T-\hat{T}}}{1-\delta}+\delta^{T-\hat{T}}\left(\left(q_{B}-q_{A}\right) \frac{1-(2 \delta)^{\hat{T}}}{1-2 \delta}+\beta\right)-2 \beta
$$

In each case, the losing platform earns zero profits.

The main qualitative results of Lemma 1 are the following. First, the same platform wins the market in all periods, so the nonfocal platform wins the market in the first period or never. Second, nonfocal platform $B$ wins the market only if it has quality advantage. Yet, platform A may win because it has a quality advantage, or it can win despite offering lower quality, because it started with a focal position. The latter occurs when $0<q_{B}-q_{A}<\beta(1-2 \delta) /\left(1-(2 \delta)^{T}\right)$, and results in an inefficient outcome. In all other cases, the higher-quality platform wins and so the equilibrium outcome is 
efficient. Observe that $\beta(1-2 \delta) /\left(1-(2 \delta)^{T}\right)$ is decreasing in $T$ and $\delta$, thus the set of parameters under which the equilibrium outcome is inefficient, decreases as $T$ and $\delta$ increase - that is, as the future becomes more important to the platforms.

Thus, competition over multiple periods yields an efficient equilibrium outcome for parameters under which in a one-period model, the lower-quality platform would win. In this sense, there is less inefficiency when the time horizon increases. One might therefore suppose that inefficiency would disappear altogether if the time horizon were extended to infinity. However that is not always the case, as the following proposition illustrates. In the proposition, we extrapolate the equilibrium outcome in Lemma 1 to the case where $T \rightarrow \infty$. For this purpose it is important to recognize that the ratio $\left(1-(2 \delta)^{T}\right) /(1-2 \delta)$ converges to $1 /(1-2 \delta)$ for $\delta<1 / 2$, and approaches infinity for $\delta>1 / 2$.

Proposition 1 (Subgame perfect equilibrium extrapolated for $T \rightarrow \infty$ ) AsT goes to infinity, the equilibrium eventually takes the following form.

(i) If $\left|q_{A}-q_{B}\right|<\beta(1-2 \delta)$ or $q_{A}=q_{B}$ then platform $A$ wins every period because it is initially focal, and its total profit converges to

$$
\frac{q_{A}-q_{B}}{1-2 \delta}+\beta
$$

(ii) Otherwise the higher quality platform wins every period and the profit of platform A converges to

$$
\Pi_{A}=\frac{q_{A}-q_{B}+\beta}{1-\delta} \text { if } q_{A}>q_{B}
$$

while the profit of platform $B$ converges to

$$
\Pi_{B}=\frac{q_{B}-q_{A}+\beta}{1-\delta}-2 \beta \text { if } q_{A}<q_{B}
$$

The losing platform earns zero profits.

[Figure 1 here]

Figure 1 illustrates which platform is active in equilibrium, depending on the parameters. It shows that the outcome of a subgame perfect equilibrium may be inefficient no matter how long the time horizon. When $0<q_{B}-q_{A}<\beta(1-2 \delta)$, platform $A$ wins despite lower quality even under infinite time horizon. That being said, the problem of inefficiency due to excessive inertia arises less often as the time horizon increases 
in length. Furthermore, the inefficiency disappears if platforms care enough about the future - that is, when $\delta>1 / 2$.

Thus, as we find in this section, the market can correct an inefficient miscoordination on the wrong platform when the consumers' expectations depend on history. In line with the general intuition, the efficient outcome emerges when platforms are sufficiently forward looking, the quality gap is sufficiently wide, and the game is played for a sufficient number of periods. One could point out, however, that in our motivating examples (i.e., the markets for smartphones, videogames, etc) we can observe inefficiency even if these conditions are satisfied. We have developed our results and the general intuition for a finite horizon environment. When it is not known when the last period will occur, it is more suitable to model the environment as an infinite horizon competition. In the rest of the paper, we analyze the infinite horizon game, and we identify two factors that may give rise to inefficient equilibria when platforms are forward looking.

\section{Markov perfect equilibria under infinite time hori- ZOn}

In Proposition 1, we characterized an equilibrium of the infinite game by extrapolating the subgame perfect equilibrium of an arbitrary finite game. If the time horizon is infinite, there may be other equilibria as well. In this section we identify Markov perfect equilibria in the infinite game ${ }^{15}$

Although the subgame perfect equilibrium identified in Proposition 1 is a Markov perfect equilibrium, there exist other Markov perfect equilibria that cannot be found by extrapolating any finite-game solution. These new equilibria often result in inefficient outcomes for the same parameters under which the equilibrium of Proposition 1 is efficient.

Every period $t$ of the infinite game is characterized by the identity $f_{t}$ of the focal platform, the state variable for that period. A Markov perfect equilibrium is characterized by the strategies of each platform in all possible states and by the outcome in each state. There can be only three pure-strategy equilibrium outcomes in a Markov equilibrium: (i) platform $A$ wins in both states, (ii) platform $B$ wins in both states,

\footnotetext{
${ }^{15} \mathrm{As}$ a further restriction, we assume that in any state, the losing platform does not choose a price that would generate negative discounted profits in case it wins the market, for instance due to unexpected demand shock.
} 
and (iii) the focal platform wins in both states 16

Next we characterize the strategies that support those equilibrium outcomes and identify the range of parameters under which each equilibrium exists. We define the value function $V_{i}^{f}$ as the equilibrium expected discounted profit of platform $i$ when platform $f$ is focal.

For our concern about excess inertia, the most interesting equilibrium outcome is when platform $A$ wins in both states. In this equilibrium, the value function for platform $B$ is $V_{B}^{B}=V_{B}^{A}=0$ because no customer joins that platform. Platform $B$ sets price $p_{B}^{f}=0$, because it has no interest in winning with price $p_{B}<0$, given that it cannot count on future profits to justify the investment required to capture the market. When $A$ is focal, it optimally sets $p_{A}^{A}=q_{A}-q_{B}+\beta$. Similarly, if platform $B$ is focal then $A$ sets price $p_{A}^{B}=q_{A}-q_{B}-\beta$ and $B$ sets $p_{B}^{B}=0$; if platform $A$ were to set a higher price, then platform $B$ would maintain its market dominance and secure nonnegative profits. Notice that as in the finite case, buyers only account for their current payoff because an individual buyer cannot affect the identity of the focal platform. Platforms, however, take into account the effect of their current prices on the future focal position, because platforms can convince all buyers to switch from one platform to the other.

We therefore have

$$
V_{A}^{A}=q_{A}-q_{B}+\beta+\delta V_{A}^{A} \quad \text { and } \quad V_{A}^{B}=q_{A}-q_{B}-\beta+\delta V_{A}^{A}
$$

yielding:

$$
V_{A}^{A}=\frac{q_{A}-q_{B}+\beta}{1-\delta} \quad \text { and } \quad V_{A}^{B}=\frac{q_{A}-q_{B}-(1-2 \delta) \beta}{1-\delta}
$$

Moreover, incentive compatibility for platform $A$ requires that $V_{A}^{A} \geq \delta V_{A}^{B}$ and $V_{A}^{B} \geq 0$. It follows that this equilibrium exists whenever $q_{A}-q_{B} \geq \beta(1-2 \delta)$. After a similar analysis for platform $B$, we arrive at our next lemma.

Lemma 2 There is an equilibrium in which platform $i$ wins in both states if and only if $q_{i}-q_{j} \geq \beta(1-2 \delta)$. The equilibrium prices are then $p_{i}^{i}=q_{i}-q_{j}+\beta, p_{i}^{j}=q_{i}-q_{j}-\beta$, $p_{j}^{j}=p_{j}^{i}=0$.

According to Lemma 2, a nonfocal platform $B$ can capture the focal position and maintain it in all future periods provided $q_{B}-q_{A} \geq \beta(1-2 \delta)$. This inequality holds

\footnotetext{
${ }^{16}$ There is no equilibrium that supports the fourth possible pure-strategy outcome - namely, that the nonfocal platform wins in both states.
} 
when $B$ 's quality is substantially higher than that of $A$, when platforms are very forward looking (so that $\delta$ is high), or when network effects $(\beta)$ are weak. We remark that, for $\delta>1 / 2$, the condition's right-hand side becomes negative, which means that an initially nonfocal platform $B$ could win the market in every period even when $q_{B}<q_{A}$. Such an equilibrium involves excess momentum and is inefficient.

Similarly, platform $A$ can dominate the market forever when $\delta>1 / 2$, even if $q_{A}<q_{B}$, as long as $q_{A}-q_{B} \geq \beta(1-2 \delta)$. This equilibrium involves excess inertia and is inefficient as well.

When $\delta=\frac{1}{2}$, an equilibrium in which platform $i$ wins exists if and only if $q_{i} \geq q_{j}$, regardless of $\beta$. Intuitively, for a nonfocal platform $i$ to win, it needs to subsidize consumers for the loss of $\beta$ in the current period (recall that $p_{i}^{j}=q_{i}-q_{j}-\beta$ ), and gains from focality in all future periods $\frac{\delta}{1-\delta} \beta$ (recall that $p_{i}^{i}=q_{i}-q_{j}+\beta$ ). When $\delta=\frac{1}{2}$, $\frac{\delta}{1-\delta}=1$, and the first subsidy equals the future benefits from focality. Consequently, $\delta=\frac{1}{2}$ is a special case in which $\beta$ does not matter: the condition for the equilibrium depends only on the quality gap, $q_{i}-q_{j}$.

It is only when the time horizon is infinite that both equilibria with excess momentum and equilibria with excess inertia occur. The nature of these equilibria can be understood as follows. Suppose that both platforms expect $A$ to behave aggressively in the future - in particular, to regain the focal position if it is ever lost. Then platform $B$ has no reason to sacrifice profit in the current period because it expects no future gain from being focal. Faced with a weak competitor, will platform $A$ decide to be aggressive as expected? If $A$ is focal then the answer is clearly Yes. But suppose that $A$ is not focal. Then the choice facing platform $A$ is this: Should it make a sacrifice today (by setting its price $p_{A}=q_{A}-q_{B}-\beta$ ) in order to gain the benefit of network effects $\beta$ in the next period, or should it wait and make that sacrifice tomorrow? If the discount factor is high enough, then the platform will opt for the sacrifice today. Hence, the beliefs that platform $A$ always win are fulfilled. Such an equilibrium therefore involves a particular form of coordination failure among firms that does not arise in the finite-horizon game, even as $T \rightarrow \infty$, because such an aggressive strategy becomes not credible as the game's end approaches.

One may wonder why platform $B$ stays in the market if it doesn't expect to sell in at least one state. One possibility is that platform $B$ is selling in some other markets (it is common for platforms to be active in several markets at different prices), allowing it to recover fixed costs. We also show in the online Appendix that our conclusion that a platform may win focality in any state despite inferior quality does not rely 
on the other platform never selling. There we show how to extend the analysis to heterogenous consumer preferences and that an equilibrium may exist where platform $A$ dominates the market in all states while platform $B$ also obtains some positive profit on the equilibrium path.

The remaining equilibrium to consider is one where the focal platform wins. Recall that $p_{i}^{f}$ denotes the price of platform $i$ when $f$ is focal in such an equilibrium. Since the winning platform anticipates that it will be focal from the new period onward, we have value functions

$$
V_{i}^{i}=\frac{p_{i}^{f}}{1-\delta} \quad \text { and } \quad V_{i}^{j}=0
$$

The benefit of selling at a given date is $p_{i}^{f}+\delta V_{i}^{i}$. It follows that the minimal profit that platform $i$ is willing to sacrifice today in order to capture the market is $-\delta V_{i}^{i}$. In such an equilibrium, the focal platform sets a price $p_{i}^{i} \leq q_{i}-q_{j}+\beta-\delta V_{j}^{j}$ because otherwise the competing platform would set a price above $-\delta V_{i}^{i}$ and win the market. Ruling out cases where $p_{j}<-\delta V_{j}^{j}$ because winning at this price would not be profitable for firm $j{ }^{17}$ we obtain the equilibrium prices

$$
p_{i}^{i}=q_{i}-q_{j}+\beta-\delta V_{j}^{j} \quad \text { and } \quad p_{j}^{i}=-\delta V_{j}^{j}
$$

In this equilibrium, the value functions solve the following equations:

$$
\begin{aligned}
& (1-\delta) V_{A}^{A}+\delta V_{B}^{B}=q_{A}-q_{B}+\beta, \\
& (1-\delta) V_{B}^{B}+\delta V_{A}^{A}=q_{B}-q_{A}+\beta,
\end{aligned}
$$

yielding

$$
V_{A}^{A}=\frac{q_{A}-q_{B}}{1-2 \delta}+\beta \quad \text { and } \quad V_{B}^{B}=\frac{q_{B}-q_{A}}{1-2 \delta}+\beta .
$$

We can therefore draw the following conclusion:

Lemma 3 There is an equilibrium where the focal platform wins in every state if and only if $\beta|1-2 \delta| \geq\left|q_{B}-q_{A}\right|$. Equilibrium prices are $p_{i}^{i}=(1-\delta) V_{i}^{i}$ and $p_{i}^{j}=-\delta V_{i}^{i}$.

We arbitrarily designated $A$ as the first period's focal platform; hence, for equilibria in which the focal platform wins in every state, platform $A$ wins every period.

For $\delta<1 / 2$ this equilibrium is the limit of the finite horizon equilibrium identified in Proposition 1 where platform $A$ maintains its focal position even when its quality is

\footnotetext{
${ }^{17}$ Allowing prices $p_{j}<-\delta V_{j}^{j}$ would not alter the existence conditions, but only the equilibrium profits.
} 
lower than that of platform $B$. Suppose now that $q_{B}$ increases; that increase has two positive effects on $V_{B}^{B}$. First, a direct effect is that, since $p_{B}^{B}=q_{B}-q_{A}+\beta-\delta p_{A}^{B}$, taking $V_{A}^{A}$ as given, platform $B$ can attract customers with a higher price $p_{B}^{B}$; this implies that $V_{B}^{B}$ will increase. Second, a strategic effect is that, since $p_{B}^{A}=-\delta V_{B}^{B}$, platform $A$ knows that even when it is focal, it will compete against a more aggressive platform $B$ because the latter would gain more by capturing focality from $A$. This threat reduces $V_{A}^{A}$, which in turn increases $V_{B}^{B}$ because a nonfocal $A$ will not compete aggressively to capture the focal position. When $\delta<1 / 2$, both the direct effect and the strategic effect increase $V_{B}^{B}$ while reducing $V_{A}^{A}$.

When $\delta>1 / 2$, the equilibrium has several unusual features. First the focal platform obtains the higher profit when it is of the lower quality. Moreover a platform's profit declines when its quality improves. In particular: as $q_{B}$ increases, platform $A$ reduces its price $p_{A}^{B}$ because it anticipates that, after becoming the focal platform, it will benefit from competing with a more efficient rival, another unusual feature of this equilibrium. We remark that if $\delta>1 / 2$, a simple within period iterated best-response dynamics would result in convergence to an equilibrium described in Lemma 2 , In this sense, we can say that, when $\delta>1 / 2$, an equilibrium where the focal platform wins in every state is unstable. The same cannot be said either for this equilibrium under $\delta \leq 1 / 2$ or, with any value of $\delta$, for the equilibria described in Lemma 2 .

Our next proposition summarizes the results of Lemmas 2 and 3.

Proposition 2 (Markov perfect equilibria) Suppose that platform $A$ is focal in period $t=1$. Then:

(i) for $q_{B}-q_{A}>\beta|1-2 \delta|$, there exists a unique equilibrium in which platform $B$ wins;

(ii) for $q_{B}-q_{A}<\beta(1-2 \delta)$, platform $A$ wins in all equilibria;

(iii) if $\delta>1 / 2$ and $\beta(1-2 \delta)<q_{B}-q_{A}<\beta(2 \delta-1)$, there exist multiple equilibria and, of these, there is one in which platform $B$ wins, and others in which $A$ wins.

[Figure 2 here]

Figure 2 illustrates which platform is active in equilibrium, depending on the parameters. It shows that, if both the discount factor and the quality differential are low, then there is a unique equilibrium in which the focal platform $A$ wins. Intuitively, 
for those parameter values the results of the dynamic game are the same as in the static game. For a positive quality differential $q_{B}-q_{A}$ and intermediate values of $\delta$, there is a unique equilibrium in which the most efficient platform wins the market and maintains its position thereafter. However, for high discount factors and low quality differential there exist multiple equilibria: some in which $A$ wins and some in which $B$ wins. Observe that disregarding the Lemma 3 equilibria (because they are unlikely to emerge) would not restore efficiency of the equilibrium in this parameter region; the reason is that there are also two equilibria, including one in which the low-quality platform wins, identified by Lemma 2. In both of these equilibria, one platform expects to encounter low competitive pressure while the other renounces capturing the market because it expects to encounter high competitive pressure - and these expectations are self-fulfilling. In sum: if the discount factor is high, then each firm's prospect of capturing the focal position is not enough to outweigh the firms' (self-fulfilling) expectations of the competitive pressure each will face.

The results of this section suggest that in our motivating examples, even if platforms are forward looking, the dominant platform may not necessarily be the platform that consumers perceive as the one with the highest quality. In the battle for dominancy in the market for smartphone operating systems or the market for videogames, our results indicate that the platform with the lowest market share may not necessary be the one that offers a poor base-quality. More importantly, market dominance may not be related to the platforms' base-quality even when platforms are forward looking.

\section{The distinction between network effects and switch- ing costs}

Network effects in our model may appear, at first blush, to play the same role as switching costs. In both cases, a consumer pays a cost when switching from one platform to the other, which can be either direct switching costs or indirect through the loss of network effects when switching to the nonfocal platform. This raises the question of what is the qualitative difference between switching costs and network effects, and how our results depend on our focus on network effects.

In this section we abstract from network effects by developing an alternative model in which $\beta$ serves as switching costs (we return to studying network effects in the next section). Platforms can price-discriminate between existing and switching consumers. We show that in a one-period game, switching costs have the same consequences as 
focality with network effects and the equilibrium is identical to the one-period game in our base model. However, in a dynamic game with infinite horizon, the main results of Section 4 no longer hold in a model with only switching costs instead of network effects 18 Therefore, the main conclusion of Section 4 is the result of network effects and cannot be replicated with switching costs only.

Consider two platforms, $A$ and $B$, that offer a base utility or quality, $q_{A}$ and $q_{B}$, where $q_{B}>q_{A}$. We abstract from network effects by assuming that there is one buyer 19 If the buyer joined a platform in period $t$, he incurs a cost $s$ when switching to the other platform at $t+1$, where $s<q_{A}<q_{B}{ }^{20}$ At the beginning of the game, the buyer is "born" on platform $A$. This is equivalent to our base-model assumption that platform $A$ is focal initially ${ }^{21}$ Let $p_{i}^{f}$ denote the price of platform $i=\{A, B\}$ when the buyer is on platform $f$ and therefore has to pay $s$ to switch from $f$ to the competing platform.

Consider first a one-period game. In an equilibrium in which platform $A$ wins, platform $B$ charges $p_{B}^{A}=0$, and platform $A$ charges $p_{A}^{A}$ such that: $q_{A}-p_{A}^{A}=q_{B}-s-p_{B}^{A}$. Hence, platform $A$ earns $p_{A}^{A}=s-\left(q_{B}-q_{A}\right)>0$ and there is an equilibrium in which platform $A$ wins iff $s>q_{B}-q_{A}$. This condition is identical to the condition for the focal platform $A$ winning under network effects, i.e., $\beta>q_{B}-q_{A}$. In what follows, suppose that $s>q_{B}-q_{A}$.

Consider now an infinitely repeated game. As before, at the beginning of $t=1$ the buyer is "born" on platform $A$. The buyer and the two platforms discount future payoffs by $\delta$.

Let's first consider a putative equilibrium equivalent to the Markov equilibrium under network effects in which platform $A$ wins in all periods whether it is "focal" or not. In the context of switching costs, platform $A$ attracts the buyer in every period regardless of whether the buyer starts the period on platform $B$ and needs to pay $s$ to switch to $A$, or the buyer starts the period on platform $A$. Define $V_{i}^{f}$ and $U^{f}$ as the discounted sum of payoffs of platform $i$ and the buyer, respectively, when the buyer is on platform $f$.

In equilibrium, platform $B$ charges $p_{B}^{B}=p_{B}^{A}=0$. The price of platform $A$ when

\footnotetext{
${ }^{18}$ In the supplementary material we show that the results hold qualitatively in environments with both network effects and small switching costs.

${ }^{19}$ This is equivalent to assuming that platforms can price discriminate between new and old buyers.

${ }^{20}$ The assumption $s<q_{A}$ ensures that consumers gain positive utilities.

${ }^{21}$ Also in the terminology of Biglaiser, Crémer and Dobos (2013)
} 
the buyer starts the period on platform $A$, i.e., $p_{A}^{A}$, solves ${ }^{22}$

$$
q_{A}-p_{A}^{A}+\delta U^{A}=q_{B}-p_{B}^{A}-s+\delta\left(q_{A}-p_{A}^{B}-s+\delta U^{A}\right)
$$

The intuition for this condition is that in equilibrium, both platforms expect that even if platform $B$ will win the incumbency position (that is, convince the buyer to switch from $A$ to $B$ ), it will not be able to maintain it in the next period. Therefore, the buyer expects that if he will stay on platform $A$, he will earn $q_{A}-p_{A}^{A}$ at the current period and then continue to stay with platform $A$ in the next period and earn $U^{A}$ (the left hand side). If however the buyer switches (the right hand side), the buyer earns $q_{B}-p_{B}^{A}-s$ in the current period. In the next period, platform $B$ will have an incumbency advantage on this particular buyer but the prices will be such that the buyer will find it optimal to switch back to platform $A$ and will earn $q_{A}-p_{A}^{B}-s+\delta U^{A}$.

In the case the buyer starts a period on platform $B$, platform $A$ sets such $p_{A}^{B}$ to attract the buyer, i.e., $p_{A}^{B}$, solves

$$
q_{A}-p_{A}^{B}-s+\delta U^{A}=q_{B}-p_{B}^{B}+\delta\left(q_{A}-p_{A}^{B}-s+\delta U^{A}\right) .
$$

The value functions given the prices are defined by

$$
V_{A}^{A}=\frac{p_{A}^{A}}{1-\delta}, \quad V_{A}^{B}=p_{A}^{B}+\delta V_{A}^{A} \quad \text { and } \quad U^{A}=\frac{q_{A}-p_{A}^{A}}{1-\delta}
$$

Substituting the prices into the value functions yields:

$$
V_{A}^{A}=-\frac{q_{B}-q_{A}}{1-\delta}+s, \quad V_{A}^{B}=-\frac{q_{B}-q_{A}}{1-\delta}-s \quad \text { and } \quad U^{A}=\frac{q_{B}}{1-\delta}-s
$$

Since $q_{B}>q_{A}$, the value $V_{A}^{B}$ is negative. Thus, under switching cost there is no equilibrium in which platform $A$ wins in both states. This result differs from the network effect case, where such an equilibrium exists for $q_{B}-q_{A}<\beta(2 \delta-1)$; we summarize the results at the end of this section.

Similarly, the value functions in the putative equilibrium in which platform $B$ wins every period, whether it starts as an incumbent or not, are

$$
V_{B}^{B}=\frac{q_{B}-q_{A}}{1-\delta}+s, \quad V_{B}^{A}=\frac{q_{B}-q_{A}}{1-\delta}-s \quad \text { and } \quad U^{B}=\frac{q_{A}}{1-\delta}-s .
$$

\footnotetext{
${ }^{22}$ Notice that the buyer anticipates that the price that will prevail next period depends on the current choice.
} 
Indeed, there is an equilibrium in which platform $B$ wins in both states if and only if $q_{B}-q_{A}>s(1-\delta)$. In comparison with the threshold under network effects, i.e., $q_{B}-q_{A}>\beta(1-2 \delta)$, under switching costs the parameter space that supports the equilibrium in which platform $B$ wins is smaller.

Next, consider a putative equilibrium equivalent to the Markov equilibrium under network effects in which the focal platform wins. In such a putative equilibrium, prices solve

$$
p_{i}^{j}=-\delta V_{i}^{i} \quad \text { and } \quad q_{i}-p_{i}^{i}+\delta U^{i}=q_{j}-p_{j}^{i}-s+\delta U^{j}
$$

Since market expectations are that the incumbent platform wins, these expectations also apply to the buyer. Therefore the buyer expects that if he switches from platform $A$ to $B$, then the next period equilibrium prices will be such that he will find it optimal to stay with platform $B$ forever.

Substituting the prices into the value functions yields

$$
V_{i}^{i}=\frac{p_{i}^{i}}{1-\delta}=\frac{q_{i}-q_{j}}{1-\delta}+s, \quad U^{i}=\frac{q_{i}-p_{i}^{i}}{1-\delta}=\frac{q_{j}}{1-\delta}-s
$$

Therefore, such an equilibrium exists if and only if $q_{B}-q_{A}<s(1-\delta)$. We summarize these findings in the following observation.

Observation In the environment with only switching costs, i.e., $\beta=0$ and $s>$ $q_{B}-q_{A}>0$ :

(i) if $q_{B}-q_{A}<s(1-\delta)$, there is a unique Markov equilibrium in which platform $A$ wins in all periods because it has the incumbency advantage at $t=1$;

(ii) if $q_{B}-q_{A}>s(1-\delta)$, there is a unique Markov equilibrium in which platform $B$ wins in all periods because it has a quality advantage.

[Figure 3 here]

Figure 3 illustrates these results. Comparing this figure with Figure 2 shows that there are two differences between switching costs and network effects. First, the region in which platform $A$ can win the market even when platforms are forward looking (the region where $\beta>\frac{1}{2}$ and $0<q_{B}-q_{A}<\beta(2 \delta-1)$ ) vanishes under switching costs. Therefore, the main result of Section 4 - that platform $A$ can exploit network effects in order to keep the focal position if $\delta$ is high - no longer holds if we reinterpret network effects as switching costs. The second difference is that the cutoff value of 
$q_{B}-q_{A}$ from which onward platform $B$ wins shift upwards from $q_{B}-q_{A}=\beta(1-2 \delta)$ under network effects to $q_{B}-q_{A}=s(1-\delta)$ under switching costs.

Here we highlight these two differences between network effects and switching costs in a model with $s>q_{B}-q_{A}>0$ and $\beta=0$. In an online appendix, we illustrate these two differences in a model with both $\beta>0$ and $s \geq 0$. We find that starting with $s=0$, an increase in $s$ has two effects. First, consistent with the first difference, the region in which platform $A$ can win the market even when platforms are forward looking decreases with $s$. Second, the cutoff value of $q_{B}-q_{A}$ from which onward platform $B$ wins shifts upwards as $s$ increases, which is consistent with the second difference between network effects and switching costs.

The intuition for difference between network effects and switching costs is the following. It is possible to think of network effects as endogenous switching costs that other buyers inflict on an individual buyer. If all other buyers switch (stay), an individual buyer "pays" switching costs if he stays (switches). As a consequence, with network effects, a buyer cannot affect the identity of the focal platform in the next period, which depends on market beliefs. In contrast, the regular switching costs are costs that the buyer inflicts on himself whenever the buyer chooses to switch from one platform to the other, regardless of the decisions of other buyers. Under switching costs the buyer can determine the identity of the platform with the incumbency advantage over himself in next period.

Therefore, replacing network effects with switching costs affects our results in two ways. First, they eliminate the equilibria that arise due to excess momentum or strong excess inertia. These equilibria depend on the buyers' beliefs concerning the participation of other buyers, which do not play a role in the absence of network effects. This result highlights the role that network effects and consumer beliefs play in shaping the market outcome, and illustrates why it is important to study network effects in isolation from the standard switching costs. The second effect of switching costs is that they increase the threshold in the quality gap between the two platforms, from which onward platform $B$ wins the market. Intuitively, with switching costs, the buyer is less willing to switch from platform $A$ to platform $B$, as doing so grants platform $B$ with an incumbency advantage over the buyer. 


\section{$6 \quad$ Stochastic qualities}

The analysis so far focused on the case where the qualities of the two platforms are constant for an infinite time horizon. We saw that, in any equilibrium, the same platform wins the market in all periods. In many markets for platforms we observe a shift in leadership every few years that results from improvements in technology. Rapid changes in leadership may give the impression that such markets are competitive and therefore "work", in sense that the platform with the best technological advancement dominates. The main goal of this section is to investigate if this is indeed the case.

In this section we consider the case in which platform qualities stochastically change between periods. We demonstrate the existence of an equilibrium in which each platform has a positive probability of winning in each period. Our main finding is that when the distribution of qualities is dispersed, there is a unique Markov equilibrium. In this equilibrium, the best platform does not always win, and the probability that the wrong platform wins may increase with $\delta$ and consequently social welfare may decline with $\delta$.

Suppose that the quality of each platform changes randomly from one period to the next. At the beginning of each period, each platform observes the realization of both its own quality and its competitor's quality for that particular period. Then the two platforms compete by setting prices.

We have shown in the previous sections that the equilibrium depends not on the absolute value of each platform's quality but rather on the difference between them. Hence we suppose, without loss of generality, that $Q \equiv q_{B}-q_{A}$ changes randomly in each period - with full support on the real line - according to a probability function $f(Q)$ with cumulative distribution function $F(Q)$. Our assumption of an infinite support ensures that there will be an equilibrium in which each platform can win the market with a positive probability ${ }^{23}$ Suppose that $Q$ has a mean $\mu>0$ such that, on average, platform $B$ is of higher quality than platform $A$; the case of $\mu<0$ is symmetric.

Let $\bar{Q}^{A}$ and $\bar{Q}^{B}$ denote equilibrium cut-offs such that, if platform $A$ is focal in period $t$, it wins if $Q \leq \bar{Q}^{A}$ but otherwise cedes market dominance to platform $B$. Conversely, if platform $B$ is focal in period $t$, then it wins if $Q \geq \bar{Q}^{B}$ but otherwise platform $A$ wins ${ }^{24}$ This equilibrium has the feature that, when $A$ is the focal platform,

\footnotetext{
${ }^{23}$ This assumption is stronger than required because our results hold also when the support is finite, provided it is wide enough. However, assuming infinite support facilitates the analysis and allows us to avoid corner solutions.

${ }^{24}$ It is straightforward to see that any Markov equilibrium must have this form.
} 
it will win in every period as long as $Q<\bar{Q}^{A}$. Then, once there is a realization with $Q>\bar{Q}^{A}$, platform $B$ wins the market and becomes focal. Platform $B$ will then maintain its focal position in future periods as along as $Q \geq \bar{Q}^{B}$, until there is a realization of $q$ with $Q<\bar{Q}^{B}$, such that platform $A$ wins back its focal position. The game repeats ad infinitum, with platforms "taking turns" at winning according to the realization of $Q$.

Let $V_{i}^{f}$ denote the expected value function of platform $i$ when platform $f$ is focal. To solve for the equilibrium, suppose that platform $A$ is focal in period $t$ and that the quality difference has a particular realization $Q$. The lowest price that platform $B$ is willing to charge in order to win the market is $-\delta V_{B}^{B}+\delta V_{B}^{A}$. This claim follows because $B$ will earn the expected value $V_{B}^{B}$ from becoming focal in the next period, and earn the expected $V_{B}^{A}$ from remaining nonfocal. Given the price of platform $B$, the highest price that allows platform $A$ to win the market is $p_{A}^{A}=\beta-Q-\delta V_{B}^{B}+\delta V_{B}^{A}$. Then $A$ earns $p_{A}^{A}+\delta V_{A}^{A}$ if it does indeed win (i.e., when $Q \leq \bar{Q}^{A}$ ) or $0+\delta V_{A}^{B}$ if it loses (i.e., when $\left.Q>\bar{Q}^{A}\right)$. Hence we can write

$$
V_{A}^{A}=\int_{-\infty}^{\bar{Q}^{A}}\left(\beta-q-\delta V_{B}^{B}+\delta V_{B}^{A}+\delta V_{A}^{A}\right) f(q) d q+\int_{\bar{Q}^{A}}^{\infty} \delta V_{A}^{B} f(q) d q
$$

Suppose now that platform $A$ is nonfocal. Then the lowest price that platform $B$ is willing to charge to maintain its focal position is $p_{B}^{B}=-\delta V_{B}^{B}+\delta V_{B}^{A}$. If $A$ wins, it sets $p_{A}^{B}$ that ensures that $-p_{A}^{B} \geq \beta-p_{B}^{B}+Q$, which writes as $p_{A}^{B}=-\beta-Q-\delta V_{B}^{B}+\delta V_{B}^{A}$. Then platform $A$ earns $p_{A}^{B}+\delta V_{A}^{A}$ if it wins the market (i.e., when $Q \leq \bar{Q}^{B}$ ) or $0+\delta V_{A}^{B}$ if it does not win (i.e., when $Q>\bar{Q}^{B}$ ). Therefore,

$$
V_{A}^{B}=\int_{-\infty}^{\bar{Q}^{B}}\left(-\beta-q-\delta V_{B}^{B}+\delta V_{B}^{A}+\delta V_{A}^{A}\right) f(q) d q+\int_{\bar{Q}^{B}}^{\infty} \delta V_{A}^{B} f(q) d q .
$$

The cases of $V_{B}^{B}$ and $V_{B}^{A}$ are symmetric: platform $B$ wins the market if $Q \geq \bar{Q}^{B}$ when it is focal or if $Q>\bar{Q}^{A}$ when it is not focal. Moreover, $Q$ has a positive effect on $B$ 's profit. It follows that

$$
\begin{gathered}
V_{B}^{B}=\int_{\bar{Q}^{B}}^{\infty}\left(\beta+q-\delta V_{A}^{A}+\delta V_{A}^{B}+\delta V_{B}^{B}\right) f(q) d q+\int_{-\infty}^{\bar{Q}^{B}} \delta V_{B}^{A} f(q) d q, \\
V_{B}^{A}=\int_{\bar{Q}^{A}}^{\infty}\left(-\beta+q-\delta V_{A}^{A}+\delta V_{A}^{B}+\delta V_{B}^{B}\right) f(q) d q+\int_{-\infty}^{\bar{Q}^{A}} \delta V_{B}^{A} f(q) d q .
\end{gathered}
$$

Next consider the equilibrium $\bar{Q}^{A}$ and $\bar{Q}^{B}$. The equilibrium $\bar{Q}^{A}$ is such that, for 
$Q=\bar{Q}^{A}$, a focal platform $A$ is indifferent between capturing the market or not, taking the equilibrium future value functions and the price of platform $B$ as given. That is,

$$
\beta-\bar{Q}^{A}-\delta V_{B}^{B}+\delta V_{B}^{A}+\delta V_{A}^{A}=\delta V_{A}^{B}
$$

Note that the condition for making the nonfocal platform $B$ indifferent between winning and losing is equivalent to the condition just stated. Analogously, the equilibrium $\bar{Q}^{B}$ should be such that, for $Q=\bar{Q}^{B}$, a nonfocal platform $A$ is indifferent between capturing the market or not, again taking the equilibrium future value functions and the price of platform $B$ as given. Thus,

$$
-\beta-\bar{Q}^{B}-\delta V_{B}^{B}+\delta V_{B}^{A}+\delta V_{A}^{A}=\delta V_{A}^{B}
$$

Once again, this condition is equivalent to the condition for making the focal platform $B$ indifferent between winning and losing.

The last six equations define the equilibrium values of $V_{A}^{A}, V_{A}^{B}, V_{B}^{B}, V_{B}^{A}, \bar{Q}^{A}$ and $\bar{Q}^{B}$. In our next proposition, we use these equations to derive a sufficient condition for the equilibrium values of $\bar{Q}^{A}$ and $\bar{Q}^{B}$ to be unique.

Proposition 3 (Unique solutions to $\bar{Q}^{A}$ and $\bar{Q}^{B}$ ) When $4 \beta \max _{q} f(q)<1$, there is a unique equilibrium with values of $\bar{Q}^{A}$ and $\bar{Q}^{B}$ characterized as follows:

(i) if $\delta=0$, then $\bar{Q}^{A}=\beta$ and $\bar{Q}^{B}=-\beta$;

(ii) $\bar{Q}^{A}-\bar{Q}^{B}=2 \beta$ for all $\delta$.

The condition $4 \beta \max _{q} f(q)<1$ requires that the quality gap be sufficiently dispersed and that network effects not be too high. These conditions ensure that a nonfocal platform can always overcome its competitive disadvantage provided its realized quality is sufficiently high, and that there exist unique equilibrium values of $\bar{Q}^{A}$ and $\bar{Q}^{B}$. Proposition 3 also shows that, when evaluated at $\delta=0$, the equilibrium cut-offs are $\bar{Q}^{A}=\beta$ and $\bar{Q}^{B}=-\beta$. It is intuitive that, when $\delta=0$, the equilibrium is identical to the one-period benchmark in which a focal platform wins as long as its quality advantage outweigh the value of network effects.

We now study the effects of $\delta, \beta$, and $\mu$ on the equilibrium values of $\bar{Q}^{A}$ and $\bar{Q}^{B}$. Toward that end, we make the simplifying assumption that $f(Q)$ is symmetric and unimodal around $\mu$. That is: $f(\mu+x)=f(\mu-x)$; and $f(Q)$ is weakly increasing in $Q$ 
for $Q<\mu$ and weakly decreasing in $Q$ for $Q>\mu{ }^{25}$ We also assume the uniqueness condition of Proposition 3, i.e., that $4 \beta f(\mu)<1$.

Proposition 4 (Effects of $\delta, \beta$, and $\mu$ on $\bar{Q}^{A}$ and $\bar{Q}^{B}$ ) Suppose that $f($.$) is sym-$ metric and unimodal around $\mu$ and that $4 \beta f(\mu)<1$. Then:

(i) Both $\bar{Q}^{A}$ and $\bar{Q}^{B}$ are decreasing in $\delta$, and if $F(0)<1 / 4$ then $\bar{Q}^{A}<0$ when $\delta$ is sufficiently high.

(ii) Both $\bar{Q}^{A}$ and $\bar{Q}^{B}$ are decreasing in $\mu$ (holding constant the distribution of $Q-\mu$ ).

(iii) If $\delta<1 / 2$, then $\bar{Q}^{A}$ is increasing and $\bar{Q}^{B}$ is decreasing in $\beta$; if $F(0)<1 / 4$ and $\delta$ is close to 1 , then $\bar{Q}^{A}$ is decreasing in $\beta$.

[Figure 4 here]

Figure 4 illustrates part (i) of Proposition 4. The figure reveals that an increase in $\delta$ need not increase the probability of the current period's higher-quality platform winning. To see why, consider first the case where platform $B$ is focal. Then, at $\delta=0$, the value is $\bar{Q}^{B}=-\beta$ and $\bar{Q}^{B}$ decrease with $\delta$. Therefore, as $\delta$ increases, a focal $B$ is more likely to win the market even when its quality realization is lower than platform $A$ 's, which implies that the probability of the "wrong" platform winning increases with $\delta$. Now consider the case in which $A$ is focal. Then, when $\delta$ is low, an increase in $\delta$ makes it less likely that a focal platform $A$ will be able to maintain its focal position with quality realization that is lower than that of platform $B$, because $\bar{Q}^{A}$ is decreasing in $\delta$. However, if $\delta$ is sufficiently high and if $F(0)<1 / 4$, then $\bar{Q}^{A}$ crosses the horizontal axis, thus becoming negative and falling further below 0 as $\delta$ increases. In this case, platform $A$ can lose the market even if it is focal and has higher quality realization than platform $B$ (when $\bar{Q}^{A}<Q<0$ ). Therefore, the probability of the "wrong" platform (i.e., one with lower quality realization) winning increases with $\delta$ when either $A$ or $B$ is focal.

The intuition underlying these results is as follows. Recall that a platform's expected profit depends on its current profit and the probability of maintaining its focal position in future periods. Since $\mu>0$, we know that $B$ is more likely to have higher quality realization than $A$ in future periods. As $\delta$ increases, platform $A$ takes into

\footnotetext{
${ }^{25}$ This is a sufficient but not a necessary condition for the results to follow. Those results may hold also when $f(Q)$ is neither symmetric nor unimodal - provided that $f(Q)$ places higher weights on positive than on negative values of $Q$, in which case $B$ is more likely than $A$ to be the focal platform in future periods.
} 
account that its chances of winning in future periods is lower and thus will have less incentive to compete aggressively in the current period. At the same time, platform $B$ takes into account that it is more likely to win in future periods and so will have more incentive to compete aggressively in the current period. These effects increase $B$ 's competitive advantage over $A$ even when the former has lower quality realization than the latter. If $F(0)<1 / 4$, then $\mu$ is sufficiently high for platform $B$ 's competitive advantage to prevent platform $A$ from capturing the market even when $A$ is focal and offers a higher quality than does $B$.

These considerations also account for part (ii) of the proposition. As $\mu$ increases, it becomes more likely that platform $B$ will have higher quality realization in future periods. Hence $B$ 's incentive to win in the current period increases, so both $\bar{Q}^{A}$ and $\bar{Q}^{B}$ decrease.

According to Proposition 4(iii), if $\delta$ is not too high then an increase in the strength of network effect makes it more likely that the focal platform wins. This result is similar to that in the one-period case. A stronger network effect increases the strategic advantage of being focal because it then becomes easier for the focal platform to attract consumers. But if $\delta$ is sufficiently high and if $F(0)<1 / 4$, then a stronger network effect reduces the ability of focal platform $A$ to retain its focal position. In this case we can see that an increase in the network effect increases the incentive of a nonfocal platform $B$ to capture the market, because $B$ is more likely than before to maintain its focal position, due to its higher expected quality.

We now turn our attention to social welfare. Our first question is whether social welfare is higher when platform $B$ or rather when platform $A$ is focal. Given that $B$ is expected to be of higher quality than $A$, one could expect that social welfare is higher when platform $B$ is focal. Yet according to Proposition 4 , the probability of platform $B$ winning despite platform $A$ 's higher quality realization increases with $\delta$, a fact that may well offset the previous effect.

In order to investigate this issue, we normalize $q_{A}$ to 0 and so $q_{B}=Q$. Let $\bar{W}^{i}$, $(i=A, B)$ denote the recursive expected social welfare when platform $i$ is focal in period $t$; thus

$$
\begin{aligned}
& \bar{W}^{A}=\int_{-\infty}^{\bar{Q}^{A}}\left(\beta+\delta \bar{W}^{A}\right) f(q) d q+\int_{\bar{Q}^{A}}^{\infty}\left(\beta+q+\delta \bar{W}^{B}\right) f(q) d q \\
& \bar{W}^{B}=\int_{\bar{Q}^{B}}^{\infty}\left(\beta+q+\delta \bar{W}^{B}\right) f(q) d q+\int_{-\infty}^{\bar{Q}^{B}}\left(\beta+\delta \bar{W}^{A}\right) f(q) d q .
\end{aligned}
$$


We normalize welfare and let $W^{i}=(1-\delta) \bar{W}^{i}$ denote the average expected perperiod welfare. Maximizing $W^{i}$ is equivalent to maximizing the total welfare. Our next proposition details the results from comparing $W^{A}$ with $W^{B}$.

Proposition 5 (Effect of $\delta$ on the per-period social welfare) Suppose that $f($. is symmetric and unimodal around $\mu$ and that $4 \beta f(\mu)<1$. Then the following statements hold.

(i) When evaluated at $\delta=0$, we have $W^{B} \geq W^{A}$; and $W^{A}$ is locally increasing in $\delta$ while $W^{B}$ is locally decreasing in $\delta$.

(ii) There is a cut-off value $\delta^{\prime}\left(0 \leq \delta^{\prime} \leq 1\right)$ such that $W^{B}>W^{A}$ for $\delta \in\left(0, \delta^{\prime}\right)$ and $W^{A}>W^{B}$ for $\delta \in\left(\delta^{\prime}, 1\right)$. A sufficient condition for $\delta^{\prime}<1$ is $F(0)<1 / 4$.

(iii) When evaluated at $\delta=1$, we have $W^{A}=W^{B}$.

Observe that the case where $Q$ is distributed uniformly along a finite interval is a special case of the symmetric and unimodal distribution in which $f(Q)$ is constant. In this case $\delta^{\prime}=0$, so that $W_{A}>W_{B}$ for $\delta \in(0,1)$ and $W_{A}=W_{B}$ for $\delta=0,1$.

Part (i) of the proposition states that $W_{B}$ is greater than $W_{A}$ for low values of $\delta$. In this case, the cut-offs $\bar{Q}^{A}$ and $\bar{Q}^{B}$ are close to their one-period levels: a focal platform $A$ wins if $Q<\beta$, and a focal platform $B$ wins if $Q>-\beta$. Because $Q$ is more likely to be positive than negative, welfare is maximized when $B$ starts out as the focal platform. But part (i) also shows that, for low values of $\delta, W_{B}$ is decreasing in $\delta$ whereas $W_{A}$ is increasing in $\delta$. This follows because, as platforms become more patient, it becomes more likely that a focal platform $B$ will win despite having quality realization lower than $A$, which reduces $W^{B}$. According to part (ii) of Proposition 5(ii), if platforms are sufficiently patient (i.e., if $\delta$ is sufficiently high) and if platform $B$ is significantly more likely than platform $A$ to be of higher quality $(F(0)<1 / 4)$, then social welfare is higher when $A$ is the initial focal platform because otherwise $B$ would have too much of a competitive advantage and so would win more often than it should. We remark that these results do not show that social welfare is maximized when platform $A$ is focal in all periods. Rather, they imply that — in the first period only — it is welfare maximizing to start the dynamic game with platform $A$ as focal even though platform $B$ is of higher quality on average.

The results obtained so far suggest that social welfare when platforms are impatient might be greater than when platforms are patient. For the general distribution function, however, the comparison between $W^{A}$ and $W^{B}$ evaluated at $\delta=0$ and $\delta=1$ 
is inconclusive. In an online appendix, we show that under a uniform distribution, social welfare when $\delta=1$ is lower than when $\delta=0$ (i.e., $\left.W^{A}\right|_{\delta=0}=\left.W^{B}\right|_{\delta=0}>\left.W^{A}\right|_{\delta=1}=$ $\left.\left.W^{B}\right|_{\delta=1}\right)$. As for consumer surplus, on the one hand an increase in $\delta$ creates the inefficiency described above: the "wrong" platform is more likely to win. On the other hand, patient platforms tend to compete more aggressively by setting lower prices. This second effect increases consumer surplus. The overall effect of $\delta$ on consumer surplus is therefore inconclusive. The online appendix shows that with a uniform distribution, consumer surplus in increasing with $\delta$ when $\mu$ is close to zero. Intuitively, in this case the first effect vanishes (as both platforms have the same expected quality) and the second effect decreases prices and hence increases consumer surplus.

This section may explain changes in leadership and evaluate their effects on welfare. The observation that the markets for smartphones and videogame consoles witnessed changes in leadership alongside with technological leaps may give the impression that such markets are efficient. However, the main conclusion if this section is that when the beliefs dynamics that we consider is at place, the low-quality platform in a given generation may win. More importantly, the probability that the wrong platform wins may increase the more platforms care about the future, resulting in a decrease in welfare. For business strategy, this result indicates that given the belief structure of our model, positive expectations concerning future quality provides a platform with a substantial competitive advantage, that may outweigh the current low quality. The platform that is expected to be high quality in the future finds it less costly to win the dominant position today. Such expectation decreases the incentive of the platform's competitors to fight aggressively and win the focal position today, as the competitors do not expect to maintain the focal position in the future.

\section{Conclusions}

In platform competition, offering the highest-quality product may not be enough to dominate the market. When there are network externalities, a platform's success depends not only on quality but also on consumers' beliefs that other consumers will join it. In a static model, a focal platform that has such a beliefs advantage may dominate the market despite offering lower quality; the result is an inefficient equilibrium. We ask whether this inefficiency can be eliminated in a dynamic game with a long time horizon.

In a model with long but finite time horizon we find that, indeed, the better 
platform wins and the efficient outcome is achieved when the future matters. More specifically, a higher-quality entrant can overcome the incumbent's network effect advantage. The future matters when both the time horizon is long and the discount factor is high. But if the discount factor is low, platforms are less concerned about the future and so the competition more nearly resembles a static game; in this case, inefficiency may persist even for a time horizon extended to infinity. We conclude that for a finite horizon social welfare is (weakly) increasing in the extent to which platforms are forward-looking, because forward orientation makes it more likely that consumers will be served by the higher-quality platform. Intuitively, a high-quality platform has more to gain by being focal in the game's final period than does the low-quality platform, which means that it will have more incentive to compete aggressively in early periods toward the end of capturing (or retaining) the focal position.

Once we modify the model to capture more realistic features, we find new sources of inefficiency even if the discount factor is high. A finite time horizon entails that platforms know when the last period occurs; if that is not known, then it is better to model it as an infinite horizon. Markov equilibria in the infinite game replicate those in the finite-horizon game extended to infinity. For high discount factors, however, additional and inefficient Markov equilibria arise in which the lower-quality platform dominates the market in all periods. These inefficient equilibria do not emerge in an alternative model in which switching costs replace network effects, because then each buyer's decision does not relay on the beliefs regarding the decisions of other buyers.

We also consider a scenario where the platforms' qualities change stochastically from period to period, which allows each platform to win any period with some probability. Here, the more the platforms are forward looking the less likely it is that even a high-quality platform will overcome its nonfocal position. This is because, if one platform is of higher quality on average than the other, then dynamic considerations give it more incentive (than in a static setting) to capture the market or to maintain its focal position, even if in the current realization it has lower quality. At the extreme, it is possible for a focal platform with higher quality realization to lose its dominance - provided that platforms are sufficiently forward looking. This finding indicates that, when qualities are stochastic, social welfare may decline as platforms become less myopic.

Our paper considers homogeneous consumers, which raises the question of how these results might be affected by the presence of heterogeneous consumers. When consumers differ in their valuations for different platforms, a focal position becomes 
less important for consumers. Armstrong (2006), for example, considers a continuum of consumers that differ in their preferences for two competing platforms. He shows that if the two platforms are sufficiently horizontally differentiated then for given platforms' prices, there is a unique allocation of consumers, such that each platform has a positive market share. Jullien and Pavan (2014) reach the same conclusion assuming that there is enough dispersion in beliefs about platforms' ability to attract consumers. Halaburda and Yehezkel (2016) show that the qualitative futures of focality follows to an extension with loyal and non-loyal consumers. Yet, the importance of focality is decreasing in the extent to which consumers are loyal to a specific platform.

Applying the intuition behind these three papers, it is reasonable to expect that increasing consumer heterogeneity reduces the effect of focality on platform profits; hence platforms will be less inclined, in that case, to compete in the current period so as to secure a future focal position.

Of course, real-life consumers are heterogeneous. Nevertheless, our motivating examples reveal that, in many markets for platforms, an important role is played by consumers' coordination problems and by platform focality. As this paper addresses the effect of dynamic considerations on the focal position of platforms, our assumption of homogeneous consumers provides us with a tractable model for determining the net effect of that market position. Moreover we show in the online appendix that our key finding that inefficient equilibria where the same platform wins the market for any history can be extended to cases with heterogeneous demand.

Our model also abstracts both from the presence of an installed base. Many markets with network effects are influenced by this factor, which constitute an additional force capable of driving excess inertia and resulting in an equilibrium where the lowerquality platform dominates for extended periods. Nonetheless, we abstract from installed base so that we can highlight the role of coordination problem as a driving force of excess inertia. While any market that exhibits network effects is affected not only by customer expectations but also by installed base, those markets are not all affected to the same extent. In the market for video-game consoles, for example, excess inertia is indeed likely driven by consumer expectations. New generations of the platforms are clearly distinguished from the previous ones by technological jumps, and backward compatibility seldom has limited appeal. In other markets, such as smartphones and computer operating systems, an installed base may play a more important role. These differences may explain the more frequent leadership changes observed in the market for video-game consoles compared with the market for computer operating systems. 
Even so, expectations and thus focality affect the market dynamics for the latter types of environments, too. We have demonstrated that, independently of other factors, it may lead to excess inertia and hence to reduced social welfare. 


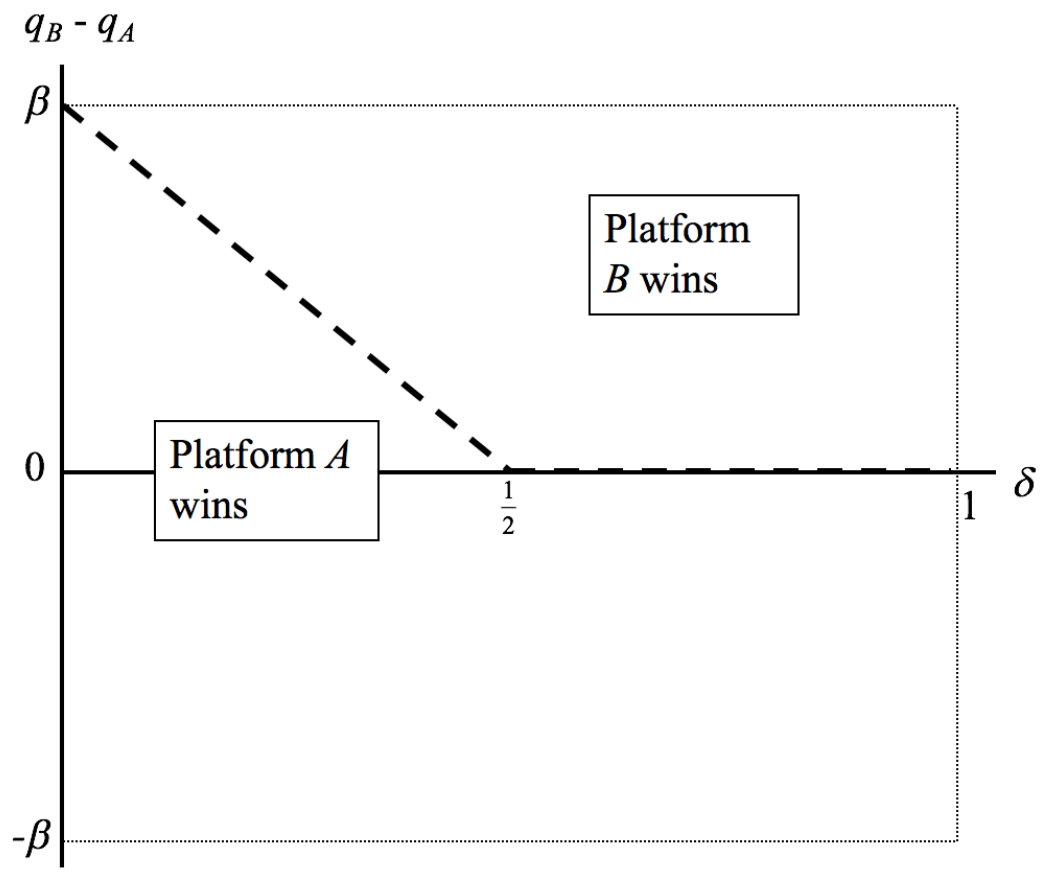

Figure 1: Active platform in subgame perfect equilibrium when $T \rightarrow \infty$ 


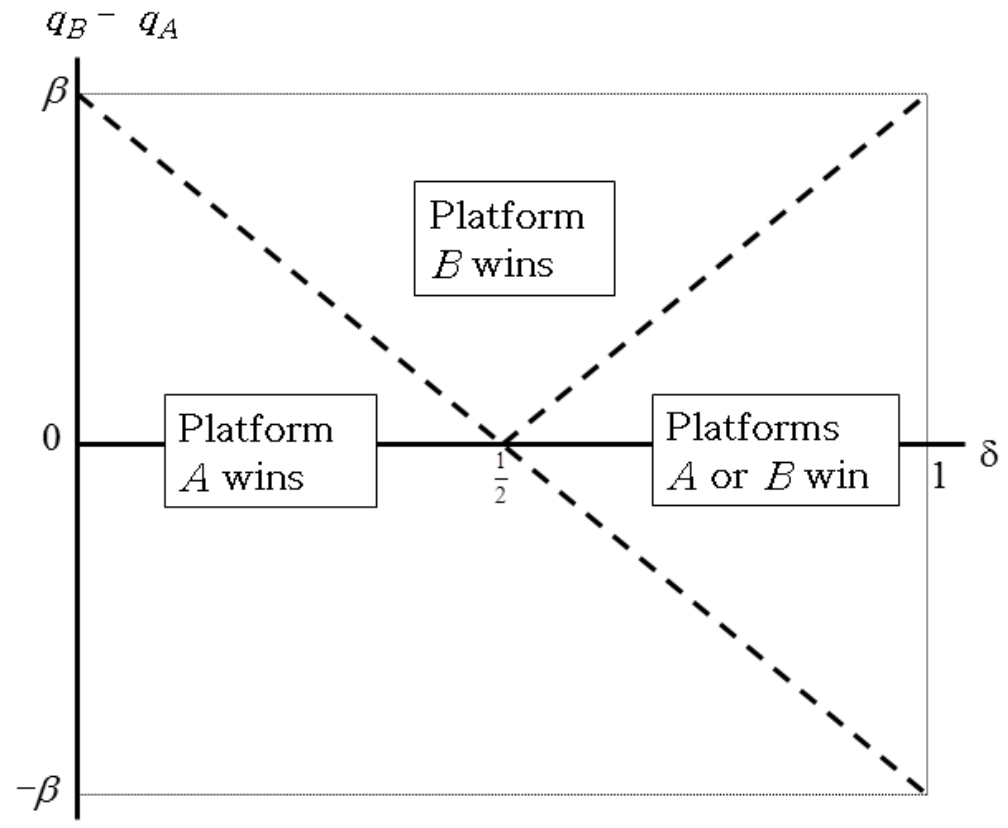

Figure 2: Equilibrium configuration 


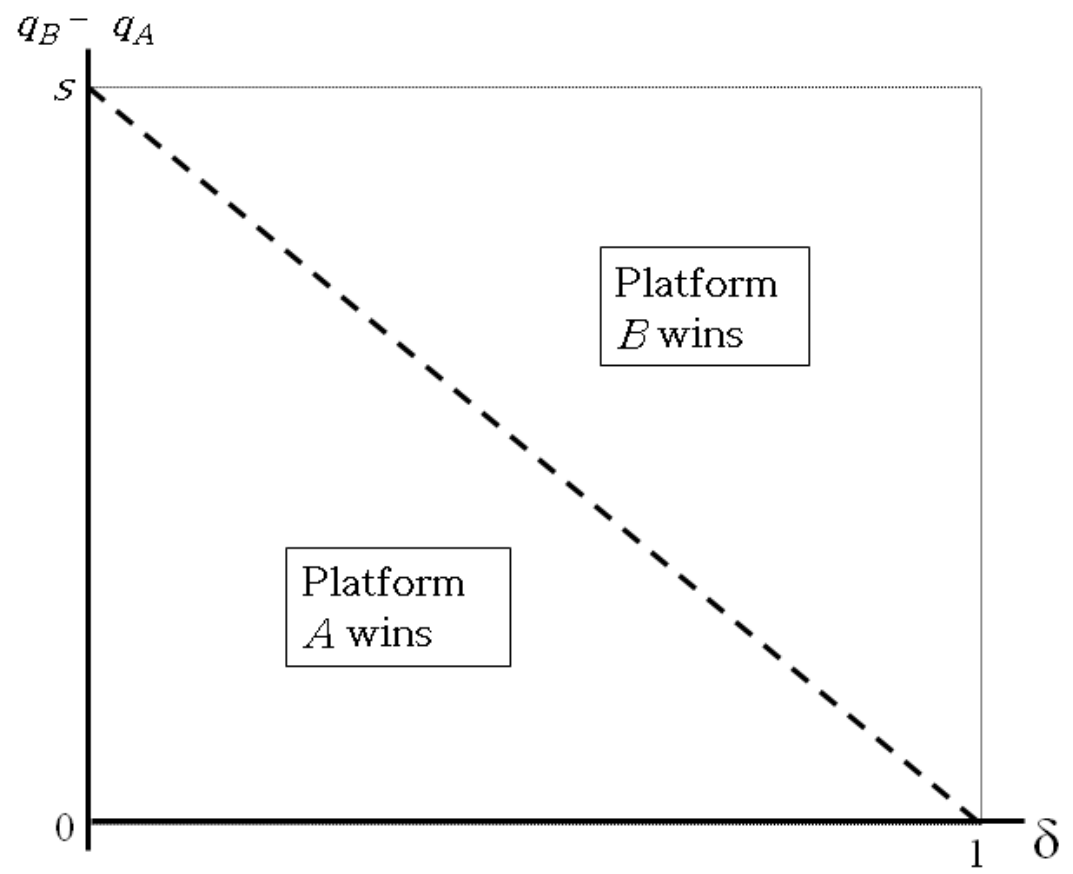

Figure 3: Equilibrium configuration with switching costs 


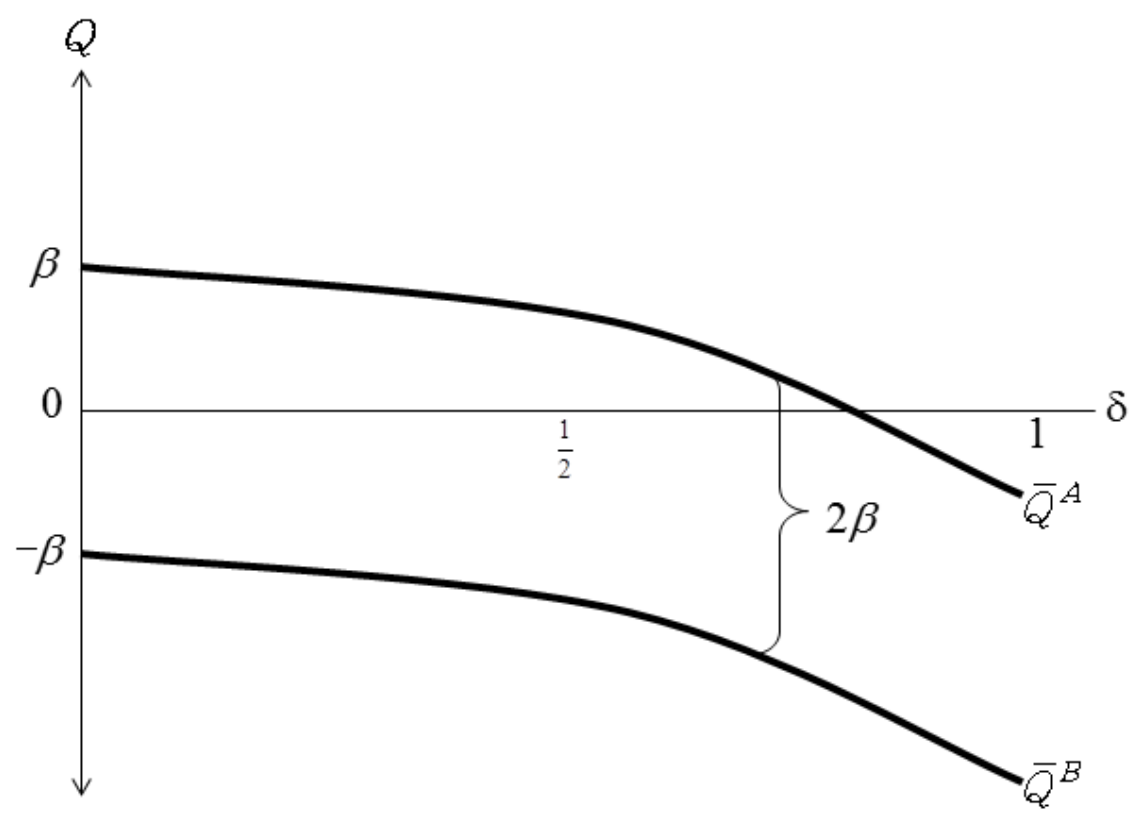

Figure 4: Effect of $\delta$ on $\bar{Q}^{A}$ and $\bar{Q}^{B}$ when $F(0)<1 / 4$ 


\section{Appendix}

\section{Proof of Lemma 1}

Let $\Pi_{i}^{f}(T)$ be the total discounted profit of platform $i$ when platform $f$ is focal at date $t=1$ and there are $T$ periods. And let $p_{i}^{f}(t)$ be the price set by platform $i$ in period $t$ when platform $f$ is focal during this period.

To win in $t=1$, the focal platform $A$ needs to set $p_{A}^{A}(1) \leq p_{B}^{A}(1)+q_{A}-q_{B}+\beta$, and set such $p_{A}^{A}(1)$ that would force $p_{B}^{A}(1) \leq-\delta \Pi_{B}^{B}(T-1)$. That is, platform $A$ wins when it sets

$$
p_{A}^{A}(1) \leq p_{B}^{A}(1)+q_{A}-q_{B}+\beta=-\delta \Pi_{B}^{B}(T-1)+q_{A}-q_{B}+\beta,
$$

in which case it earns

$$
\Pi_{A}^{A}(T \mid A \text { wins in } t=1)=q_{A}-q_{B}+\beta-\delta \Pi_{B}^{B}(T-1)+\delta \Pi_{A}^{A}(T-1) .
$$

Notice that calculated in such a way the profit under the condition of winning may be negative. Then, the optimal action is to cede the market and earn no profit. Therefore, the profit from unconditionally optimal actions is $\Pi_{i}^{f}(T)=\max \left\{\Pi_{i}^{f}(T \mid i\right.$ wins in $t=$ 1), 0$\}$. Using similar logic,

$$
\begin{aligned}
\Pi_{B}^{A}(T \mid B \text { wins in } t=1) & =q_{B}-q_{A}-\beta-\delta \Pi_{A}^{A}(T-1)+\delta \Pi_{B}^{B}(T-1) \\
& =-\Pi_{A}^{A}(T \mid A \text { wins in } t=1) .
\end{aligned}
$$

Let $\hat{\Pi}_{i}^{f}(T) \equiv \Pi_{i}^{f}(T \mid i$ wins in $t=1)$. Then $\Pi_{i}^{f}(T)=\max \left\{\hat{\Pi}_{i}^{f}, \delta \Pi_{i}^{j}(T-1)\right\}$. Notice that $\Pi_{i}^{f}(T)$ is bounded from below by 0 , while $\hat{\Pi}_{i}^{f}(T)$ is not.

Suppose that $\hat{\Pi}_{i}^{i}(k)>0$ for both $i=A, B$ and $k=1, \ldots, T-1$. Then from (6) we obtain 26

$$
\begin{aligned}
\hat{\Pi}_{i}^{i}(T) & =q_{i}-q_{j}+\beta-\delta \hat{\Pi}_{j}^{j}(T-1)+\delta \hat{\Pi}_{i}^{i}(T-1) \\
& =\left(q_{i}-q_{j}\right) \sum_{k=1}^{T}(2 \delta)^{k-1}+\beta=\left(q_{i}-q_{j}\right) \frac{1-(2 \delta)^{T}}{1-2 \delta}+\beta .
\end{aligned}
$$

\footnotetext{
${ }^{26}$ This follows from applying the same formulas recursively in

$$
\hat{\Pi}_{i}^{i}(T-1)-\hat{\Pi}_{j}^{j}(T-1)=2\left(q_{i}-q_{j}\right)+2 \delta\left[\hat{\Pi}_{i}^{i}(T-2)-\hat{\Pi}_{j}^{j}(T-2)\right]=2\left(q_{i}-q_{j}\right) \sum_{k=1}^{T-1}(2 \delta)^{k-1} .
$$
}


The fraction $\frac{1-(2 \delta)^{T}}{1-2 \delta}$ is positive and increasing with $T$. Therefore, $\hat{\Pi}_{i}^{i}(T)$ is also monotonic. When $q_{i}-q_{j}>0$, then $\hat{\Pi}_{i}^{i}(T)$ is positive and increasing. Conversely, when $q_{i}-q_{j}<0$ then $\hat{\Pi}_{i}^{i}(T)$ is decreasing and when $q_{i}-q_{j}<-\beta \frac{1-2 \delta}{1-(2 \delta)^{T}}$, it may even be negative ${ }^{27}$ And once it is negative, it stays negative for all larger $T$.

Now, suppose $\left(q_{i}-q_{j}\right) \frac{1-(2 \delta)^{T}}{1-2 \delta}+\beta<0$. By the monotonic properties of $\frac{1-(2 \delta)^{T}}{1-2 \delta}$, it may only happen for $q_{i}<q_{j}$, and there exists $T_{i} \leq T$ such that $\left(q_{i}-q_{j}\right) \frac{1-(2 \delta)^{T_{i}}}{1-2 \delta}+\beta<0$ and either $\left(q_{i}-q_{j}\right) \frac{1-(2 \delta)^{T_{i}-1}}{1-2 \delta}+\beta>0$ or $q_{i}-q_{j}+\beta<0$. In the latter case, $T_{i}=1$. That is, $T_{i}$ is the shortest time horizon for which it is not worth capturing the market. For time horizon $T_{i}$ and shorter, $\hat{\Pi}_{i}^{i}(T)$ for $i=A, B$ can be calculated using (8) — but not for longer horizons.

In what follows we make use of the following intermediate result:

Intermediate claim: If $\hat{\Pi}_{i}^{i}(T)<0$ then, for all $T^{\prime}>T, \hat{\Pi}_{i}^{i}\left(T^{\prime}\right)<0$.

Proof of the intermediate claim. Suppose $T_{i}>1$. By definition of $T_{i}, \hat{\Pi}_{i}^{i}\left(T_{i}-1\right)>$ 0 (and given by (8)), and

$$
\hat{\Pi}_{i}^{i}\left(T_{i}\right)=q_{i}-q_{j}+\beta-\delta \hat{\Pi}_{j}^{j}\left(T_{i}-1\right)+\delta \hat{\Pi}_{i}^{i}\left(T_{i}-1\right)<0 .
$$

Now $\hat{\Pi}_{i}^{i}(T)$ for $T>T_{i}$ can no longer be calculated using (8). We need to apply (7) directly:

$$
\hat{\Pi}_{i}^{i}\left(T_{i}+1\right)=q_{i}-q_{j}+\beta-\delta \Pi_{j}^{j}\left(T_{i}\right)+\delta \Pi_{i}^{i}\left(T_{i}\right)=q_{i}-q_{j}+\beta-\delta \hat{\Pi}_{j}^{j}\left(T_{i}\right)
$$

since $\Pi_{j}^{j}\left(T_{i}\right)=\hat{\Pi}_{j}^{j}\left(T_{i}\right)$ and $\Pi_{i}^{i}\left(T_{i}\right)=0$.

By properties of (8), $\hat{\Pi}_{j}^{j}\left(T_{i}\right)>\hat{\Pi}_{j}^{j}\left(T_{i}-1\right)$. From $\hat{\Pi}_{i}^{i}\left(T_{i}\right)<0$, we have $\delta \Pi_{j}^{j}\left(T_{i}-1\right)>$ $q_{i}-q_{j}+\beta+\delta \hat{\Pi}_{i}^{i}\left(T_{i}-1\right)>q_{i}-q_{j}+\beta$. Thus $\delta \hat{\Pi}_{j}^{j}\left(T_{i}\right)>q_{i}-q_{j}+\beta$ and $\hat{\Pi}_{i}^{i}\left(T_{i}+1\right)<0-$ and so forth for each $T>T_{i}$.

This completes the proof of the intermediate claim.

Thus, for $T>T_{i}, \Pi_{i}^{i}(T)=0$. Moreover, $\Pi_{j}^{j}(T)=\hat{\Pi}_{j}^{j}(T)$ also can no longer be

\footnotetext{
${ }^{27}$ This also implies that one of the $\Pi_{i}^{i}(T)$ must be positive. A negative $\hat{\Pi}_{i}^{i}(T)$ for some $T$ implies $q_{i}-q_{j}<0$, and $q_{j}-q_{i}>0$ implies $\hat{\Pi}_{j}^{j}(T)>0$ for all $T$.
} 
calculated using (8). Applying (7) directly:

$$
\begin{aligned}
& \Pi_{j}^{j}\left(T_{i}+1\right)=q_{j}-q_{i}+\beta+\delta \Pi_{j}^{j}\left(T_{i}\right), \\
& \Pi_{j}^{j}\left(T_{i}+2\right)=q_{j}-q_{i}+\beta+\delta\left(q_{j}-q_{i}+\beta\right)+\delta^{2} \Pi_{j}^{j}\left(T_{i}\right) .
\end{aligned}
$$

More generally, for any $T>T_{i}$ we have

$$
\begin{aligned}
\Pi_{j}^{j}(T) & =\left(q_{j}-q_{i}+\beta\right) \sum_{t=1}^{T-T_{i}} \delta^{t-1}+\delta^{T-T_{i}} \Pi_{j}^{j}\left(T_{i}\right) \\
& =\left(q_{j}-q_{i}+\beta\right) \frac{1-\delta^{T-T_{i}}}{1-\delta}+\delta^{T-T_{i}}\left(\left(q_{j}-q_{i}\right) \frac{1-(2 \delta)^{T_{i}}}{1-2 \delta}+\beta\right) .
\end{aligned}
$$

Notice that for the case when $T_{i}=1, \Pi_{j}^{j}(T)$ reduces to $\left(q_{j}-q_{i}+\beta\right) \frac{1-\delta^{T}}{1-d}$.

Now, using those properties of $\Pi_{i}^{i}(T)$, for $i=A, B$, we can consider following cases.

(i) $\left|q_{A}-q_{B}\right|<\beta \frac{1-2 \delta}{1-(2 \delta)^{T}}$

Then both $\hat{\Pi}_{A}^{A}(k)$ and $\hat{\Pi}_{B}^{B}(k)$ are positive for all $k=1, \ldots, T$. Since platform $A$ is focal in $t=0$ and $\hat{\Pi}_{A}^{A}(T)$ is positive, the platform never cedes the market and its profit is $\Pi_{A}^{A}(T)=\hat{\Pi}_{A}^{A}(T)=\left(q_{A}-q_{B}\right) \frac{1-(2 \delta)^{T}}{1-2 \delta}+\beta$ (by (8)).

(ii a) $q_{A}-q_{B}>\beta \frac{1-2 \delta}{1-(2 \delta)^{T}}$

That is, $\left(q_{B}-q_{A}\right) \frac{1-(2 \delta)^{T}}{1-2 \delta}+\beta<0$, and thus, by the arguments above, there exists a $\hat{T}=T_{B}<T$. This means that $\hat{\Pi}_{B}^{B}(T)<0$; that is, platform $B$ would not find it worthwhile to win the market even if it was focal, given $A$ 's quality advantage. Platform $A$ wins the market, but the prices it charges and profit depend on $T_{B}$, as derived earlier:

$$
\Pi_{A}^{A}(T)=\left(q_{A}-q_{B}+\beta\right) \frac{1-\delta^{T-T_{B}}}{1-\delta}+\delta^{T-T_{B}}\left(\left(q_{A}-q_{B}\right) \frac{1-(2 \delta)^{T_{B}}}{1-2 \delta}+\beta\right) .
$$

(ii b) $q_{B}-q_{A}>\beta \frac{1-2 \delta}{1-(2 \delta)^{T}}$

Now there exists a $\hat{T}=T_{A}<T$. That is, $\hat{\Pi}_{A}^{A}(T)<0$; in other words, it is not worthwhile for platform $A$ to defend the market in $t=1$, given the quality advantage of platform $B$. Then platform $B$ wins the market in $t=1$, becomes the focal platform and keeps the market for the rest of the time horizon. To win the market, in $t=1$, platform $B$ sets $p_{B}^{A}(1)=q_{B}-q-A-\beta$, while platform $A$ sets $p_{A}^{A}(1)=0$. In the next period, platform $B$ is the focal platform with quality advantage and with $T-1$ period time horizon. Thus, the discounted total profit 
is as that of platform $A$ in case (ii), with relabeling the platforms and length of the time horizon. That is:

$$
\begin{aligned}
\Pi_{B}^{A}(T) & =q_{B}-q_{A}-\beta+\delta\left[\left(q_{B}-q_{A}+\beta\right) \frac{1-\delta^{T-1-T_{A}}}{1-\delta}+\delta^{T-1-T_{A}}\left(\left(q_{A}-q_{B}\right) \frac{1-(2 \delta)^{T_{A}}}{1-2 \delta}+\beta\right)\right] \\
& =\left(q_{B}-q_{A}+\beta\right) \frac{1-\delta^{T-T_{A}}}{1-\delta}+\delta^{T-T_{A}}\left(\left(q_{B}-q_{A}\right) \frac{1-(2 \delta)^{T_{A}}}{1-2 \delta}+\beta\right)-2 \beta .
\end{aligned}
$$

This completes the proof of Lemma 1 .

\section{Proof of Proposition 1}

The proof follows immediately from extrapolating the outcome of Lemma 1 to $T \rightarrow \infty$.

\section{Proof of Lemma 2}

For an allocation where the platform $i$ wins in both states to be an equilibrium, it is both necessary and sufficient that $V_{i}^{i}=\frac{q_{i}-q_{j}+\beta}{1-\delta} \geq \delta V_{i}^{j}=\delta \frac{q_{i}-q_{j}+(2 \delta-1) \beta}{1-\delta} \geq 0$ which gives the condition. Moreover there is no other equilibrium where platform $i$ wins in both states because such an equilibrium requires that firm $j$ prices are zero in both states, implying unique best-reply prices for platform $i$.

This completes the proof of lemma 2 .

\section{Proof of Lemma 3}

For an allocation where the focal platform wins in every state to be an equilibrium, it is both necessary and sufficient that $V_{A}^{A} \geq 0$ and $V_{B}^{B} \geq 0$. Given our restriction that a platform does not choose a price that would be unprofitable if it won the market, the equilibrium is uniquely defined by the above conditions.

\section{Proof of Proposition 2}

The Lemma 1 result that the same platform wins in all periods (for constant quantities $q_{A}$ and $q_{B}$ ) holds for infinite game as well. Therefore, there are no other equilibria than those in which either platform $i$ or a focal platform always wins.

Under the condition of (i), Lemma 2 implies that the only equilibrium outcome is one where platform $B$ wins in both states and this equilibrium is unique. The condition of (ii) rules out the possibility that platform $B$ wins in both states, hence the result 
follows from our assumption that $A$ is initially focal. Under the condition of (iii), Lemmas 2 and 3 identify 3 equilibria: either the same platform wins in both states or the focal platform wins in each states.

The following table summarizes the result:

\begin{tabular}{|c|c|c|c|}
\hline & $\begin{array}{c}\text { (i) } \\
q_{B}-q_{A}>\beta|1-2 \delta|\end{array}$ & $\begin{array}{c}\text { (ii) } \\
q_{B}-q_{A}<\beta(1-2 \delta)\end{array}$ & $\begin{array}{c}\text { (iii) } \\
\beta(1-2 \delta)<q_{B}-q_{A}<\beta(2 \delta-1)\end{array}$ \\
\hline $\begin{array}{l}\delta<1 / 2 \\
|1-2 \delta|=1-2 \delta\end{array}$ & $\begin{array}{c}q_{B}-q_{A}>\beta(1-2 \delta) \\
B \text { wins by Lem } 2\end{array}$ & $\begin{array}{l}\left|q_{B}-q_{A}\right|<\beta(1-2 \delta) \\
\text { and } A \text { wins by Lem } 3 \text {, } \\
\text { or } q_{A}-q_{B}>\beta(1-2 \delta) \\
\text { and } A \text { wins by Lem } 2\end{array}$ & $\emptyset$ \\
\hline $\begin{array}{l}\delta>1 / 2 \\
\begin{aligned}|1-2 \delta| & =2 \delta-1 \\
& >1-2 \delta\end{aligned}\end{array}$ & $\begin{array}{c}q_{B}-q_{A}>\beta(1-2 \delta) \\
B \text { wins by Lem } 2\end{array}$ & $\begin{array}{l}\left|q_{B}-q_{A}\right|<\beta|1-2 \delta| \\
\text { and } A \text { wins by Lem } 3 \\
\text { or } q_{A}-q_{B}>\beta(1-2 \delta) \\
\text { and } A \text { wins by Lem } 2\end{array}$ & $\begin{array}{l}\text { because } \beta(1-2 \delta)<q_{B}-q_{A} \\
\quad B \text { wins by Lem } 2 \\
\text { because } q_{A}-q_{B}>\beta(1-2 \delta) \\
\quad A \text { wins by Lem } 2 \\
\text { because }\left|q_{B}-q_{A}\right|<\beta|1-2 \delta| \\
\quad A \text { wins by Lem } 3\end{array}$ \\
\hline
\end{tabular}

\section{Proof of Proposition 3}

Directly from the formulas for $V_{A}^{A}, V_{A}^{B}, V_{B}^{B}, V_{B}^{A}$, and conditions for $\bar{Q}^{A}$ and $\bar{Q}^{B}$, we obtain

$$
\bar{Q}^{A}-\bar{Q}^{B}=2 \beta
$$

Moreover,

$$
\begin{aligned}
V_{A}^{A} & =\int_{-\infty}^{\bar{Q}^{A}}\left(\bar{Q}^{A}-q\right) f(q) d q+\delta V_{A}^{B} \\
V_{A}^{B} & =\int_{-\infty}^{\bar{Q}^{B}}\left(\bar{Q}^{B}-q\right) f(q) d q+\delta V_{A}^{B}=\frac{1}{1-\delta} \int_{-\infty}^{\bar{Q}^{B}}\left(\bar{Q}^{B}-q\right) f(q) d q
\end{aligned}
$$

and

$$
\begin{aligned}
V_{B}^{B} & =\int_{\bar{Q}^{B}}^{+\infty}\left(q-\bar{Q}^{B}\right) f(q) d q+\delta V_{B}^{A}, \\
V_{B}^{A} & =\frac{1}{1-\delta} \int_{\bar{Q}^{A}}^{+\infty}\left(q-\bar{Q}^{A}\right) f(q) d q .
\end{aligned}
$$


The optimality condition is then

$$
\bar{Q}^{A}=\beta-\delta V_{B}^{B}+\delta V_{B}^{A}+\delta V_{A}^{A}-\delta V_{A}^{B}
$$

which can be rewritten as

$$
\bar{Q}^{A}=\beta+\delta \phi\left(\bar{Q}^{A}\right)
$$

where

$$
\begin{aligned}
\phi\left(\bar{Q}^{A}\right)= & \int_{\bar{Q}^{A}}^{+\infty}\left(q-\bar{Q}^{A}\right) f(q) d q+\int_{-\infty}^{\bar{Q}^{A}}\left(\bar{Q}^{A}-q\right) f(q) d q \\
& -\int_{-\infty}^{\bar{Q}^{B}}\left(\bar{Q}^{B}-q\right) f(q) d q-\int_{\bar{Q}^{B}}^{+\infty}\left(q-\bar{Q}^{B}\right) f(q) d q .
\end{aligned}
$$

Integrating by parts yields

$$
\phi\left(\bar{Q}^{A}\right)=-2 \beta+2 \int_{\bar{Q}^{A}-2 \beta}^{\bar{Q}^{A}} F(q) d q .
$$

Now we have

$$
\begin{aligned}
\phi^{\prime}\left(\bar{Q}^{A}\right) & =2\left(F\left(\bar{Q}^{A}\right)-F\left(\bar{Q}^{A}-2 \beta\right)\right), \\
\phi(-\infty) & =-2 \beta \\
\phi(+\infty) & =2 \beta
\end{aligned}
$$

These properties imply that $\bar{Q}^{A}>\beta+\delta \phi\left(\bar{Q}^{A}\right)$ for $\bar{Q}^{A}=\infty$ and that $\bar{Q}^{A}<\beta+\delta \phi\left(\bar{Q}^{A}\right)$ for $\bar{Q}^{A}=-\infty$. Hence there is a unique solution to $\bar{Q}^{A}$ if $\bar{Q}^{A}-\beta-\delta \phi\left(\bar{Q}^{A}\right)$ is increasing in $\bar{Q}^{A}$ i.e. if $\delta \phi^{\prime}\left(\bar{Q}^{A}\right)<1$. We observe that $\delta \phi^{\prime}\left(\bar{Q}^{A}\right)<1$ when

$$
2 \delta \max _{q}(F(q)-F(q-2 \beta))<1
$$

In this case, the equilibrium is unique. This is the case for all $\delta$ and if $4 \beta \max _{q} f(q)<1$.

Finally, notice that when evaluated at $\delta=0$, the solution to $\bar{Q}^{A}=\beta+\delta \phi\left(\bar{Q}^{A}\right)$ is $\bar{Q}^{A}=\beta$.

This completes the proof of Proposition 3 


\section{Proof of Proposition 4}

Proof of part (i): Since $\bar{Q}^{A}=\beta+\delta \phi\left(\bar{Q}^{A}\right)$,

$$
\frac{\partial \bar{Q}^{A}}{\partial \delta}=\frac{\phi\left(\bar{Q}^{A}\right)}{1-\delta \phi^{\prime}\left(\bar{Q}^{A}\right)}
$$

From the proof of Proposition 3, if $4 \beta f(\mu)<1$ then $1-\delta \phi^{\prime}\left(\bar{Q}^{A}\right)>0$. To see that $\phi\left(\bar{Q}^{A}\right)<0$ for all $\bar{Q}^{A} \leq \beta$, suppose first that $\bar{Q}^{A}<\mu$. Then

$$
\phi\left(\bar{Q}^{A}\right)=-2 \int_{\bar{Q}^{A}-2 \beta}^{\bar{Q}^{A}}\left(\frac{1}{2}-F(q)\right) d q<0
$$

where the inequality follows because symmetric and unimodal distribution (SUD) implies that for all $Q<\mu, F(Q)<1 / 2$. Next, consider $\mu<\bar{Q}^{A} \leq \beta$. Then:

$$
\phi\left(\bar{Q}^{A}\right)=-2 \int_{\bar{Q}^{A}-2 \beta}^{\mu-\left(\bar{Q}^{A}-\mu\right)}\left(\frac{1}{2}-F(q)\right) d q-2 \int_{\mu-\left(\bar{Q}^{A}-\mu\right)}^{\mu+\left(\bar{Q}^{A}-\mu\right)}\left(\frac{1}{2}-F(q)\right) d q<0,
$$

where the first term is negative because $\bar{Q}^{A}>\mu>0$ and SUD implies that $F(\mu-$ $\left.\left(\bar{Q}^{A}-\mu\right)\right)<F(\mu)=\frac{1}{2}$ and the second term equals 0 because SUD implies that $F(\mu+x)-\frac{1}{2}=\frac{1}{2}-F(\mu-x)$. Since $\phi\left(\bar{Q}^{A}\right)<0$ we have $\frac{\partial \bar{Q}^{A}}{\partial \delta}<0$, and since $\bar{Q}^{B}=\bar{Q}^{A}-2 \beta$ it follows that $\frac{\partial \bar{Q}^{B}}{\partial \delta}<0$.

Next, $\bar{Q}^{A}<0$ if

$$
0>\beta+\delta \phi(0)
$$

which holds for $\delta$ large if

$$
-\beta>\phi(0)=-2 \beta(1-2 F(-2 \beta))+\int_{-2 \beta}^{0}(-2 q) f(q) d q=-2 \beta+2 \int_{-2 \beta}^{0} F(q) d q
$$

or if

$$
\beta>2 \int_{-2 \beta}^{0} F(q) d q
$$

This inequality holds for all $\beta$ if $F(0)<1 / 4$.

Proof of part (ii): Let $F(Q ; \mu)$ denote the $F(Q)$ given $\mu$. We have:

$$
\frac{\partial \bar{Q}^{A}}{\partial \mu}=\frac{2 \int_{\bar{Q}^{A}-2 \beta}^{\bar{Q}^{A}}\left(\frac{\partial F(Q ; \mu)}{\partial \mu}\right) d q}{1-\delta \phi^{\prime}\left(\bar{Q}^{A}\right)}<0
$$

where the inequality follows because SUD implies that $F(q ; \mu)$ is decreasing in $\mu$. 
Proof of part (iii): We have:

$$
\frac{\partial \bar{Q}^{A}}{\partial \beta}=\frac{1-2 \delta+4 \delta F\left(\bar{Q}^{A}-2 \beta\right)}{1-\delta \phi^{\prime}\left(\bar{Q}^{A}\right)}>0
$$

where the inequity follows because $1-2 \delta+4 \delta F\left(\bar{Q}^{A}-2 \beta\right)>0$ if $\delta<\frac{1}{2}$. Since $\bar{Q}^{B}=\bar{Q}^{A}-2 \beta$, it follows that

$$
\frac{\partial \bar{Q}^{B}}{\partial \beta}=-\left[\frac{1+\delta\left(2-4 F\left(\bar{Q}^{A}\right)\right)}{1-2 \delta\left(F\left(\bar{Q}^{A}\right)-F\left(\bar{Q}^{A}-2 \beta\right)\right)}\right]<0
$$

where the inequality follows because the numerator in brackets is positive when $\delta<$ $\frac{1}{2}$ (since $F\left(\bar{Q}^{A}\right)<1$ ) and because the denominator is positive when $\delta<\frac{1}{2}$ (since $\left.F\left(\bar{Q}^{A}\right)-F\left(\bar{Q}^{A}-2 \beta\right)<1\right)$. When $F(0)<1 / 4$ and $\delta=1$, we have

$$
\left.\frac{\partial \bar{Q}^{A}}{\partial \beta}\right|_{\delta=1}=\frac{-1+4 F\left(\bar{Q}^{B}\right)}{1-\phi^{\prime}\left(\bar{Q}^{A}\right)}<\frac{-1+4 \frac{1}{4}}{1-\phi^{\prime}\left(\bar{Q}^{A}\right)}=0,
$$

where the inequality follows because $F\left(\bar{Q}^{B}\right)<F(0)<1 / 4$.

This completes the proof of Proposition 4.

\section{Proof of Proposition 5}

Solving for $W^{A}$ and $W^{B}$, we obtain

$$
\begin{aligned}
W^{A} & =\beta+\frac{\left(1-\delta+\delta F\left(\bar{Q}^{B}\right) \int_{\bar{Q}^{A}}^{\infty} q f(q) d q+\delta\left(1-F\left(\bar{Q}^{A}\right)\right) \int_{\bar{Q}^{B}}^{\infty} q f(q) d q\right.}{1-\delta F\left(\bar{Q}^{A}\right)+\delta F\left(\bar{Q}^{B}\right)} \\
W^{B} & =\beta+\frac{\delta F\left(\bar{Q}^{B}\right) \int_{\bar{Q}^{A}}^{\infty} q f(q) d q+\left(1-\delta F\left(\bar{Q}^{A}\right)\right) \int_{\bar{Q}^{B}}^{\infty} q f(q) d q}{1-\delta F\left(\bar{Q}^{A}\right)+\delta F\left(\bar{Q}^{B}\right)}
\end{aligned}
$$

First consider $W^{A}$. Solving the derivative of $W^{A}$ with respect to $\delta$ and then evaluating at $\delta=0$ yields

$$
\begin{aligned}
\left.\frac{\partial W^{A}}{\partial \delta}\right|_{\delta=0} & =\left(1-F\left(\bar{Q}^{A}\right)\right)\left(\int_{\bar{Q}^{B}}^{\infty} q f(q) d q-\int_{\bar{Q}^{A}}^{\infty} q f(q) d q\right)-f\left(\bar{Q}^{A}\right) \bar{Q}^{A} \frac{\partial \bar{Q}^{A}}{\partial \delta} \\
& =(1-F(\beta)) \int_{-\beta}^{\beta} q f(q) d q-f(\beta) \beta \frac{\partial \bar{Q}^{A}}{\partial \delta},
\end{aligned}
$$

where the equality follows from the substitutions $\bar{Q}^{A}=\beta$ and $\bar{Q}^{B}=-\beta$. By our assumption of SUD, $\int_{-\beta}^{\beta} q f(q) d q \geq 0$ (proof available upon request), implying the the first term is nonnegative. Since Proposition 4 shows that $\bar{Q}^{A}$ is decreasing in $\delta$, the 
second term is positive implying that $\left.\frac{\partial W^{A}}{\partial \delta}\right|_{\delta=0}>0$.

Next consider $W^{B}$. Solving the derivative of $W^{B}$ with respect to $\delta$ and then evaluating at $\delta=0$, we have

$$
\begin{aligned}
\left.\frac{\partial W^{B}}{\partial \delta}\right|_{\delta=0} & =-F\left(\bar{Q}^{B}\right)\left(\int_{\bar{Q}^{B}}^{\infty} q f(q) d q-\int_{\bar{Q}^{A}}^{\infty} q f(q) d q\right)-f\left(\bar{Q}^{B}\right) \bar{Q}^{B} \frac{\partial \bar{Q}^{B}}{\partial \delta} \\
& =-F(-\beta) \int_{-\beta}^{\beta} q f(q) d q+f(-\beta) \beta \frac{\partial \bar{Q}^{B}}{\partial \delta}
\end{aligned}
$$

where the equality follows from the substitutions $\bar{Q}^{B}=-\beta$ and $\bar{Q}^{A}=\beta$. Again by our assumption of SUD, $\int_{-\beta}^{\beta} q f(q) d q \geq 0$, implying the the first term is nonpositive. Since Proposition 4 shows that $\bar{Q}^{B}$ is decreasing in $\delta$, the second term is also negative implying that $\left.\frac{\partial W^{B}}{\partial \delta}\right|_{\delta=0}<0$.

Now we consider the gap $W^{B}-W^{A}$ :

$$
W^{B}-W^{A}=\frac{(1-\delta)\left(\int_{\bar{Q}^{B}}^{\infty} q f(q) d q-\int_{\bar{Q}^{A}}^{\infty} q f(q) d q\right)}{1-\delta F\left(\bar{Q}^{A}\right)+\delta F\left(\bar{Q}^{B}\right)}=\frac{(1-\delta)}{1-\delta F\left(\bar{Q}^{A}\right)+\delta F\left(\bar{Q}^{B}\right)} M\left(\bar{Q}^{A}\right),
$$

where

$$
M\left(\bar{Q}^{A}\right)=\int_{\bar{Q}^{A}-2 \beta}^{\bar{Q}^{A}} q f(q) d q .
$$

Since $1 \geq F(q) \geq 0$ and $0 \leq \delta \leq 1$, it follows that $\operatorname{sgn}\left(W^{B}-W^{A}\right)=\operatorname{sgn}\left(M\left(\bar{Q}^{A}\right)\right)$.

Consider first $\delta=0$ such that $\bar{Q}^{A}=\beta$. Then, SUD implies $M(\beta)=\int_{-\beta}^{\beta} q f(q) d q \geq$ 0 and $W^{B}-W^{A} \geq 0$. Second, consider $\delta=1$. Then $W^{B}-W^{A}=\frac{0}{1} M\left(\bar{Q}^{A}\right)$, where $M\left(\bar{Q}^{A}\right)$ is finite; hence $W^{B}-W^{A}=0$.

Next, we turn to $1>\delta^{\prime}$. We distinguish between two case, $F(0)<1 / 4$ and $F(0)>1 / 4$, which will be analyzed in turn.

Case 1: $F(0)<1 / 4$. In this case, Proposition 4 implies that there is a cutoff, $\delta^{\prime \prime}$ where $\delta^{\prime \prime}$ is the solution to $\bar{Q}^{A}=0$, such that $\bar{Q}^{A}>0$ for $\delta \in\left[0, \delta^{\prime \prime}\right)$ and $\bar{Q}^{A}<0$ for $\delta \in\left(\delta^{\prime \prime}, 1\right]$. For all $\delta \in\left[\delta^{\prime \prime}, 1\right], M\left(\bar{Q}^{A}\right)<0$ because $Q<0$ for all $Q \in\left[\bar{Q}^{A}-2 \beta, \bar{Q}^{A}\right]$. For $\delta \in\left[0, \delta^{\prime \prime}\right), M\left(\bar{Q}^{A}\right)$ is decreasing with $\delta$. To see why:

$$
\frac{\partial M\left(\bar{Q}^{A}\right)}{\partial \delta}=\left[\bar{Q}^{A} f\left(\bar{Q}^{A}\right)-\left(\bar{Q}^{A}-2 \beta\right) f\left(\bar{Q}^{A}-2 \beta\right)\right] \frac{\partial \bar{Q}^{A}}{\partial \delta} .
$$

The term inside brackets is positive for all $\delta \in\left[0, \delta^{\prime \prime}\right]$ because $\bar{Q}^{A} \geq 0$ and $f(q)>0$ and because $\bar{Q}^{A} \leq \beta$ implies that $\bar{Q}^{A}-2 \beta \leq \beta-2 \beta=-\beta<0$. Since $\bar{Q}^{A}$ is decreasing in $\delta$, we have $\frac{\partial M\left(\bar{Q}^{A}\right)}{\partial \delta}<0$.

To summarize, $M\left(\bar{Q}^{A}\right) \geq 0$ for $\delta=0, M\left(\bar{Q}^{A}\right)$ is decreasing with $\delta$ for $\delta \in\left[0, \delta^{\prime \prime}\right)$, 
and $M\left(\bar{Q}^{A}\right)<0$ for $\delta \in\left(\delta^{\prime \prime}, 1\right]$. Hence there is a unique cutoff $\delta^{\prime}<\delta^{\prime \prime}$ such that $M\left(\bar{Q}^{A}\right)>0$ for $\delta \in\left[0, \delta^{\prime}\right)$ and $M\left(\bar{Q}^{A}\right)<0$ for $\delta \in\left(\delta^{\prime}, 1\right]$. Since $\operatorname{sgn}\left(W^{B}-W^{A}\right)=$ $\operatorname{sgn} M\left(\bar{Q}^{A}\right)$, this implies that $W_{B}>W_{A}$ for $\delta \in\left[0, \delta^{\prime}\right)$ and $W_{B}<W_{A}$ for $\delta \in\left(\delta^{\prime}, 1\right)$.

Case 2: $F(0)>1 / 4$. In this case, $\bar{Q}^{A}>0$ at $\delta=1$. Notice that $M\left(\bar{Q}^{A}\right)$ is decreasing with $\delta$ for all $\delta \in[0,1]$ (the proof that $\frac{\partial M\left(\bar{Q}^{A}\right)}{\partial \delta}<0$ requires only that $\bar{Q}^{A}>0$ which holds in Case 2 for all $\left.\delta \in[0,1]\right)$. However, unlike Case 1 , now $M\left(\bar{Q}^{A}\right)$ at $\delta=1$ can be either positive or negative. It will be positive if $\bar{Q}^{A}$ at $\delta=1$ is sufficiently higher than 0 , in which case $M\left(\bar{Q}^{A}\right)>0$ for all $\delta \in[0,1]$ and so $W_{B}>W_{A}$ for all $\delta \in[0,1)$. In this case $\delta^{\prime}=1$. Note that $M\left(\bar{Q}^{A}\right)$ can be negative at $\delta=1$ if $\bar{Q}^{A}$ at $\delta=1$ is sufficiently close to 0 , in which case at $\delta=1$; then $M\left(\bar{Q}^{A}\right)<0$ and so $W_{B}>W_{A}$ for $\delta \in\left[0, \delta^{\prime}\right)$ and $W_{B}<W_{A}$ for $\delta \in\left(\delta^{\prime}, 1\right)$, as in Case 1 .

Remark on uniform distribution. If the distribution is uniform, then $M\left(\bar{Q}^{A}\right)=0$ at $\delta=0$ and $M\left(\bar{Q}^{A}\right)<0$ otherwise. This implies that $W_{A}>W_{B}$ for all $\delta \in(0,1)$ and $W_{A}=W_{B}$ otherwise.

This completes the proof of Proposition 5.

\section{References}

[1] Akerlof, Robert, Richard Holden and Luis Rayo, "Network Externalities and Market Dominance," working paper, (2018).

[2] Argenziano, Rossella and Itzhak Gilboa, "History as a Coordination Device," Theory and Decision, Vol. 73 (2012), pp. 501-512

[3] Armstrong, Mark. "Competition in two?sided markets." RAND Journal of Economics 37.3 (2006), pp. 668-691.

[4] Austen, Ian, "BlackBerry 10 Prototype Is Given to Developers," New York Times (2012). Available at: http://www.nytimes.com/2012/05/02/technology/researchin-motions-blackberry-10-is-unveiled.html

[5] Besanko, David, Ulrich Doraszelski, and Yaroslav Kryukov. "The economics of predation: What drives pricing when there is learning-by-doing?." American Economic Review 104.3 (2014), pp. 868-897.

[6] Biglaiser, Gary, Jacques Crémer, and Gergely Dobos. "The value of switching costs." Journal of Economic Theory 148.3 (2013): 935-952. 
[7] Biglaiser, Gary, Jacques Crémer, "The value of incumbency in heterogeneous platforms," TSE Working Paper (2016).

[8] Bunton, Cam, "BlackBerry Q10 vs. iPhone 5 Hardware Comparison." Today's iPhone (2013). Available at: http://www.todaysiphone.com/2013/07/blackberryq10-vs-iphone-5-hardwarecomparison/

[9] Cabral, Luis, "Dynamic Price Competition with Network Effects," Review of Economic Studies, Vol. 78 (2011), pp. 83-111.

[10] Caillaud, Bernard and Bruno Jullien, "Competing Cybermediaries," European Economic Review, Vol. 45 (2001), pp. 797-808.

[11] Caillaud, Bernard and Bruno Jullien, "Chicken \& Egg: Competition among Intermediation Service Providers," RAND Journal of Economics, Vol. 34 (2003), pp. 309-328.

[12] Chen, Kai and Edison Tse, "Dynamic Platform Competition in Two-Sided Markets," mimeo, Stanford University, 2008.

[13] David, Paul A. "Clio and the Economics of QWERTY," American Economic Review 75.2 (1985), pp. 332-337.

[14] Doganoglu, Toker, "Dynamic price competition with consumption externalities," netnomics 5.1 (2003), pp. 43-69.

[15] Farrell, Joseph, and Paul Klemperer. "Coordination and lock-in: Competition with switching costs and network effects." Handbook of industrial organization 3 (2007): 1967-2072.

[16] Fudenberg, Drew, and Jean Tirole. "Pricing a network good to deter entry." Journal of Industrial Economics 48.4 (2000), pp. 373-390.

[17] Hagiu, Andrei, "Pricing and Commitment by Two-Sided Platforms," RAND Journal of Economics, Vol. 37 (2006), pp. 720-737.

[18] Hagiu, Andrei and Hanna Halaburda, "Responding to the Wii?" Harvard Business School Case Study No. 709-448 and Teaching Note No. 709-481 (2009).

[19] Halaburda, Hanna and Yaron Yehezkel, "Platform Competition under Asymmetric Information," American Economic Journal: Microeconomics, Vol. 5 (2013), pp. 22-68. 
[20] Halaburda, Hanna and Yaron Yehezkel, "The Role of Coordination Bias in Platform Competition," Journal of Economics and Management Strategy (2016), Vol. 25 , pp. $274-312$.

[21] Hossain, Tanjim, and John Morgan. "The quest for QWERTY." American Economic Review 99, no. 2 (2009), pp. 435-440.

[22] Jullien, Bruno, "Competition in Multi-Sided Markets: Divide-and-Conquer," American Economic Journal: Microeconomics, Vol. 3 (2011), pp. 1-35.

[23] Jullien, Bruno, and Alessandro Pavan. "Platform pricing under dispersed information." No. 1568R. Discussion Paper, Center for Mathematical Studies in Economics and Management Science (2014).

[24] Laussel, Didier, and Joana Resende. "Competition in the market vs competition for the market: The case of cell phone networks." GREQAM and CORE, November (2007).

[25] Liebowitz, Stan J., and Stephen E. Margolis. "The fable of the keys." The journal of law \& economics 33.1 (1990), pp. 1-25.

[26] Lopez, Angel and Patrick Rey, "Foreclosing Competition through Access Charges and Price Discrimination." Journal of Industrial Organization (forthcoming).

[27] Markovich, Sarit, "Snowball: A dynamic oligopoly model with indirect network effects," Journal of Economic Dynamics \& Control, Vol. 32 (2008), pp. 909-938.

[28] Mitchell, Matthew F., and Andrzej Skrzypacz. "Network externalities and longrun market shares." Economic Theory 29.3 (2006), pp. 621-648. 


\section{Supplements to "Dynamic Competition with Network Externalities: How History Matters"

\author{
by Hanna Halaburda, Bruno Jullien and Yaron Yehezkel
}

July 26, 2019

\section{Heterogeneous consumers}

In this appendix, we extend our model to show:

i) how to extend the concept of focal platform to the case of heterogenous consumers.

ii) that a Markov equilibrium may exist where a low-quality platform $A$ stays or becomes focal in all states while platform $B$ also obtains positive profits. Hence, platform $B$ has an incentive to remain active in the market even though it does not win the focal position.

\subsection{Static analysis}

Let us assume that the market stays covered but the perception of the quality differential between the two platforms varies across consumers. More precisely, consider our base model and suppose that the quality differential $q=q^{B}-q^{A}$ is heterogenous, distributed in the population (of size 1) according to a distribution $F$ on a support $(\underline{q}, \bar{q})$ which may be infinite. Then if all consumers with quality differential below $\hat{q}$ join platform $A$, the relative gain in value of joining platform $B$ is $q+\beta(1-F(\hat{q}))-\beta F(\hat{q})$. Thus, at any period, possible allocations of consumers 
are the solutions to $D_{A}=F(\hat{q})$, where $D_{A}$ is the demand for platform $A$ and ${ }^{1}$

$$
\begin{aligned}
\hat{q}+\beta(1-2 F(\hat{q})) & =p_{B}-p_{A}, \\
\text { or } \hat{q} & =\bar{q} \leq p_{B}-p_{A}+\beta, \\
\text { or } \hat{q} & =\underline{q} \geq p_{B}-p_{A}-\beta .
\end{aligned}
$$

With a general distribution $F$, for some range of prices, there may be multiple allocations of consumers that constitute consumers' best responses to the prices and strategies of other consumers (henceforth "outcomes"). The concept of focality then implies that consumers coordinate on the outcome that yields the largest demand for the focal platform.

We may then extend our analysis by assuming that in any period $t$ the platform that sells the most in the current period becomes focal in the next period.

To illustrate how focality shapes demand, suppose that the distribution of $q$ has density $f(q)$ where $f$ is continuous unimodal with a peak at $\mu>0$. The slope of $q+\beta(1-2 F(q))$ is $1-2 \beta f(q)$. Assume network effects are strong enough that $2 f(\mu) \beta>1$. Then the function $q+\beta(1-2 F(q))$ is not monotonic. More precisely, defining $q_{1}$ and $q_{2}$ as the smallest and the largest roots of

$$
1=2 \beta f\left(q_{i}\right),
$$

the function $q+\beta(1-2 F(q))$ is

$$
\begin{array}{lc}
\text { increasing on } & q<q_{1}, \\
\text { decreasing on } & q_{1}<q<q_{2}, \\
\text { increasing on } & q>q_{2} .
\end{array}
$$

We conclude that if $\Delta_{2}=q_{2}+\beta\left(1-2 F\left(q_{2}\right)\right)<p_{B}-p_{A}<\Delta_{1}=q_{1}+\beta\left(1-2 F\left(q_{1}\right)\right)$, there are three possible outcomes for the allocation of consumers. This is illustrated in Figure 1 which shows the function for a normal distribution and $\mu=1$. On the range $\left(\Delta_{2}, \Delta_{1}\right)$, the intermediate outcome is unstable. Then focality selects the allocation with $\hat{q}>q_{2}$ if platform $A$ is focal, and the allocation with $\hat{q}<q_{1}$ if platform $B$ is focal. The figure shows the respective marginal consumers.

Notice that when platform $A$ is focal (thick red curve), there is a discontinuous

\footnotetext{
${ }^{1}$ Akerlof, Holden and Rayo (2018) analyze a similar demand system.
} 


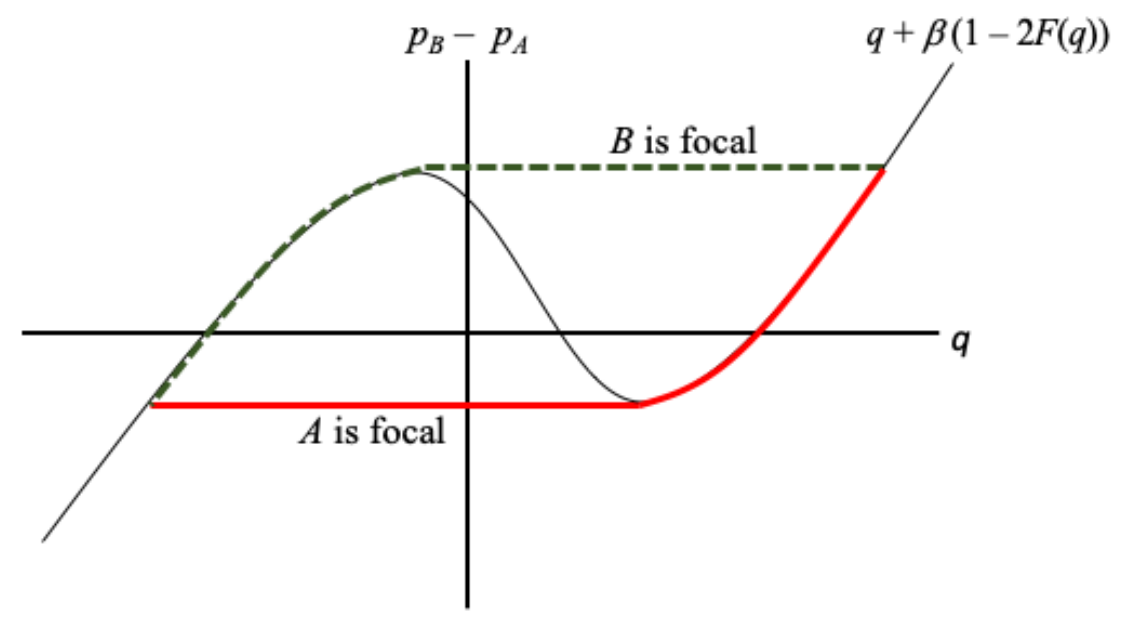

Figure 1: the function $q+\beta(1-2 F(q))$

jump in its demand at $p_{B}-p_{A}=\Delta_{2}$. Moreover, provided that $\beta$ is large or $\mu$ is small, the value of $\Delta_{2}$ is negative so that despite lower quality on average, a focal platform $A$ may sell more that its competitor at higher prices, a feature that was key in our analysis.

\subsection{Illustration}

We now use an example to illustrate how the static and the dynamic analyses extend to heterogeneous consumers.

Consider a distribution $F$ on $(\mu-1, \mu+1)$ that consists of a constant density $f<1 / 2$ and a mass point $1-2 f$ at $\mu$, where we assume that $2 \beta f<1$.

This is a limit case of a unimodal distribution when the peak goes to infinity. Then $q+\beta(1-2 F(q))$ is increasing linearly except for a downward discontinuity at $\mu$ :

$$
\begin{array}{ll}
q+\beta(1-2 F(q))=q+\beta(1-2 f)+2 \beta f(\mu-q) & \text { if } \mu-1 \leq q<\mu \\
q+\beta(1-2 F(q))=q-\beta(1-2 f)+2 \beta f(\mu-q) & \text { if } \mu<q \leq \mu+1 .
\end{array}
$$

Let us first consider a one-period game where platform $A$ is focal. Firms set prices 
$p_{A}$ and $p_{B}$, and consumers with $q<q^{A}$ join the focal platform, where:

$$
\begin{aligned}
p_{B}-p_{A} & =q^{A}+\beta(1-2 f)+2 \beta f\left(\mu-q^{A}\right) & & \text { if } \left.\mu-1+\beta<p_{B}-p_{A}<\mu-\beta(1-2 f)\right), \\
q^{A} & =\mu-1 & & \text { if } p_{B}-p_{A}<\min (\mu-1+\beta, \mu-\beta(1-2 f)) \\
p_{B}-p_{A} & =q^{A}-\beta(1-2 f)+2 \beta f\left(\mu-q^{A}\right) & & \text { if } \mu-\beta(1-2 f)<p_{B}-p_{A}<\mu+1-\beta \\
q^{A} & =\mu+1 & & \text { if } p_{B}-p_{A}>\max (\mu+1-\beta, \mu+\beta(1-2 f)) .
\end{aligned}
$$

Let us consider a candidate outcome with $D_{A}=F\left(\hat{q}^{A}\right), \mu<\hat{q}^{A}<\mu+1$ and $\hat{p}_{B}^{A}-$ $\hat{p}_{A}^{A}=\hat{q}^{A}-\beta(1-2 f)+2 \beta f\left(\mu-\hat{q}^{A}\right)$. Consider the choice of price by platform $A$. Holding the price $p_{B}$ constant, choosing $p_{A}$ in the range $\left(\hat{p}_{B}^{A}-\mu-1+\beta, \hat{p}_{B}^{A}-\mu+\beta(1-2 f)\right)$ amounts to choosing $\hat{q}$ in the range $(\mu, \mu+1)$ with the profit

$$
\Pi^{A}=\left(\hat{p}_{B}^{A}-\hat{q}+\beta(1-2 f)-2 \beta f(\mu-\hat{q})\right)(1-f+f(\hat{q}-\mu))
$$

which is concave. The first order condition for the price of $A$ is then

$$
-(1-\beta 2 f)\left(1-f+f\left(\hat{q}^{A}-\mu\right)\right)+\hat{p}_{A}^{A} f=0 .
$$

Similarly, given $\hat{p}_{A}^{A}$, choosing a price $p_{B}$ in the range $\left(\hat{p}_{A}^{A}+\mu-\beta(1-2 f), \hat{p}_{A}^{A}+\mu+1-\beta\right)$ yields concave profit

$$
\Pi^{B}=\left(\hat{p}_{A}^{A}+\hat{q}-\beta(1-2 f)+2 \beta f(\mu-\hat{q})\right) f(1+\mu-\hat{q})
$$

leading to the first-order condition

$$
(1-\beta 2 f) f\left(1+\mu-\hat{q}^{A}\right)-\hat{p}_{B}^{A} f=0 .
$$

Adding the two first-order conditions together yields

$$
-(1-\beta 2 f)\left(1-f+f\left(\hat{q}^{A}-\mu\right)\right)+(1-\beta 2 f) f\left(1+\mu-\hat{q}^{A}\right)+\left(\hat{p}_{A}^{A}-\hat{p}_{B}^{A}\right) f=0 .
$$

Using $\hat{p}_{B}^{A}-\hat{p}_{A}^{A}=\hat{q}^{A}-\beta(1-2 f)+2 \beta f\left(\mu-\hat{q}^{A}\right)$, we obtain

$$
\hat{q}^{A}=\mu+\frac{(1-2 \beta f)(2 f-1)+\beta(1-2 f) f-f \mu}{3(1-2 \beta f) f},
$$


which lies between $\mu$ and $\mu+1$ if

$$
\frac{-f-1+3 \beta f}{f}<\mu<\frac{(1-2 f)(3 f \beta-1)}{f} .
$$

This holds for a small $\mu$ if

$$
\frac{f+1}{3}>\beta f>\frac{1}{3}
$$

Prices are then

$$
\begin{aligned}
& \hat{p}_{A}^{A}=(1-2 \beta f) \frac{F\left(\hat{q}^{A}\right)}{f}=\frac{2-f(1+3 \beta+\mu)}{3 f}>0, \\
& \hat{p}_{B}^{A}=(1-\beta 2 f) \frac{1-F\left(\hat{q}^{A}\right)}{f}=\frac{1+f(1-3 \beta+\mu)}{3 f}>0,
\end{aligned}
$$

where the inequalities follow because whenever $\mu<\hat{q}^{A}<\mu+1$, then $1>F\left(\hat{q}^{A}\right)>0$. Given these prices, platform $A$ cannot profit from reducing the price below $\hat{p}_{B}^{A}-\mu-1+\beta$ as this would not raise demand above 1 . Platform $A$ cannot profit from increasing the price above $\hat{p}_{B}^{A}-\mu+\beta(1-2 f)$, because doing so would result in a discontinuous decrease in platform $A$ 's demand. ${ }^{2}$

Consider now platform $B$. It has no profitable deviation for prices above $\hat{p}_{A}+\mu-$ $\beta(1-2 f)$, because profit is concave on the relevant range. Setting $p_{B}<\hat{p}_{A}+\mu-$ $\beta(1-2 f)$ is not profitable if $\hat{p}_{A}+\mu-\beta(1-2 f)<0$, which holds for $\mu$ small if

$$
\frac{2-f}{6(1-f)}<\beta f
$$

As $\frac{2-f}{6(1-f)}>\frac{1}{3}$, we conclude that this an equilibrium for $\mu$ small if

$$
\frac{f+1}{3}>\beta f>\frac{2-f}{6(1-f)}
$$

Since $f<\frac{1}{2}$, (6) holds only when $\beta>1$, which we assume in what follows.

To conclude, we find that when the conditions (6), $f<\frac{1}{2}, \beta>1$ and $1>2 \beta f$ hold, there is a static equilibrium in which platform $A$ is focal. Prices are given by (3) and (4), and both platforms gain a positive market share. Notice that the range of parameters satisfying these conditions is nonempty.

\footnotetext{
${ }^{2}$ The optimal deviation on this range can be shown to be at $\hat{p}_{B}^{A}-\mu+\beta(1-2 f)$ which can not be optimal as $p_{A}$ slightly below this level induces an upward jump in demand.
} 


\subsection{Dynamic analysis}

Still assuming a uniform distribution with a mass-point, we can now extend our analysis to a dynamic case. In particular the next result shows that with an infinite horizon and patient firms, there exists a Markov equilibrium where platform $A$ stays focal in any state. In this equilibrium, when platform $A$ is focal in a certain period, both platforms set the static Nash prices defined in the previous section:

$$
p_{A}^{A}=\hat{p}_{A}^{A}, \quad p_{B}^{A}=\hat{p}_{B}^{A}, \quad \text { and } \quad q^{A}=\hat{q}^{A} .
$$

And both platforms gain positive market share (i.e., $\mu-1<\hat{q}^{A}<\mu+1$ ). When platform $B$ is focal in a certain period, then in equilibrium

$$
p_{A}^{B}=-\mu-\beta(1-2 f), \quad p_{B}^{B}=0, \quad \text { and } \quad \hat{q}^{B}=\mu+1 .
$$

That is, the nonfocal platform $A$ sets a negative price (recall that $f<\frac{1}{2}$ ), dominates the entire market, and becomes focal in the next period.

Notice that this equilibrium is qualitatively similar to the equilibrium in our base model. In both cases, platforms set the static prices when $A$ is focal, and platform $A$ sets a negative price when it is nonfocal. The main difference is that here, the losing platform $B$ has an incentive to remain active. When it is nonfocal, platform $B$ gains positive market share. When it is focal, platform $B$ earns zero profits in the current period — followed by positive profits in all future periods - making it worthwhile for platform $B$ to remain active.

The Markov equilibrium is characterized by $p_{i}^{j}, D_{i}^{j}, q^{j}$ and $V_{i}^{j}$, where $i$ is the platform and $j$ the focal platform. The equilibrium profit is

$$
V_{i}^{j}=p_{i}^{j} D_{i}^{j}+\delta V_{i}^{A}
$$

where $D_{A}^{j}=1-D_{B}^{j}=F\left(q^{j}\right)$. The equilibrium values are $V_{A}^{j}=p_{A}^{j} F\left(q^{j}\right)+\delta V_{A}^{A}$ and $V_{B}^{j}=p_{B}^{j}\left(1-F\left(q^{j}\right)\right)+\delta V_{B}^{A}$.

To solve for this equilibrium, suppose first that platform $A$ is focal. Platform $B$ plays its short-term best-response because it expects that even if it gains the focal position, in the next period it will earn zero profits. Platform $A$ plays its short-term best response as well, because doing so is enough to maintain its focal position. We therefore conclude that when platform $A$ is focal, the equilibrium prices are the same 
as in the static case. The value functions are

$$
V_{A}^{A}=\frac{\hat{p}_{A}^{A} F\left(\hat{q}^{A}\right)}{1-\delta}>0 \quad \text { and } \quad V_{B}^{A}=\frac{\hat{p}_{B}^{A}\left(1-F\left(\hat{q}^{A}\right)\right)}{1-\delta}>0
$$

Notice that static Nash equilibrium conditions ensure that firm $i$ would not deviate from such an equilibrium when $A$ is focal if $V_{i}^{A} \geq V_{i}^{B}$, because the deviation profit gain would be smaller than in a static game. We will see below that this is the case for both platforms.

Suppose now that platform $B$ is focal. By our assumptions that $2 f \beta<1$ and $\beta>1$, we have $\mu+\beta(1-2 f)>\mu+1-\beta$ and $\mu-1+\beta>\mu-\beta(1-2 f)$. This implies that at the equilibrium prices $p_{A}^{B}=-\mu-\beta(1-2 f)$ and $p_{B}^{B}=0, p_{B}-p_{A}>$ $\max (\mu+1-\beta, \mu+\beta(1-2 f))$, and therefore $\hat{q}^{A}=1+\mu$ and platform $A$ dominates the market. Platform $B$ would not deviate because winning the market in the current period would require a negative price and would delay by one period the time where it can sell at positive prices. Platform $B$ cannot profitably deviate to a higher price, because it will not gain positive market share. Firm $A$ could deviate by setting nonnegative price and lose focality, but the profit would be $\delta V_{A}^{B}$ which is less than $V_{A}^{B}$ and thus not profitable if $V_{A}^{B}>0$. Thus this is an equilibrium if

$$
\mu+\beta(1-2 f)<\delta \frac{\hat{p}_{A}^{A} F\left(\hat{q}^{A}\right)}{1-\delta} \Longleftrightarrow \frac{\mu+\beta(1-2 f)}{\mu+\beta(1-2 f)+\hat{p}_{A}^{A} F\left(\hat{q}^{A}\right)}<\delta<1
$$

which holds for large $\delta$.

This shows that excess inertia equilibria - where despite lower quality one platform would price aggressively and win back its focal position had it lost it - are robust to demand heterogeneity. It also shows that in this situation the nonfocal platform can survive with a positive market share.

We conclude this Appendix by pointing out that a similar reasoning would show that by contrast, for high discount factors, a Markov equilibrium where in any state the focal platform stays focal does not exist with demand heterogeneity. This confirms that this type of equilibrium should be expected only if firms are not too forward-looking. 


\section{Network Effects and Switching Costs}

This extension shows that when platforms can price-discriminate between existing and switching consumers, the results of Section 4 (existence of Markov perfect equilibria under infinite time horizon where the same platform always wins, even if it is of lower quality) also hold in a setting with both network effects and switching costs. Moreover, this section highlights how network effects and switching costs differently affect the results.

Consider our base model and suppose that consumers experience both network effects, $\beta$, and switching costs, $s$. We maintain our assumptions that $q_{A}>s \geq 0$ and $\beta \geq q_{B}-q_{A} \geq 0$. We allow platforms to price discriminate between existing and new consumers. And we explore the existence of Markov equilibria where on the equilibrium path all consumers buy from the same firm (which becomes focal). For consistency with our base model, we distinguish between the equilibrium and out-ofequilibrium prices. Consider the out-of-equilibrium scenario in which all consumers are on a focal platform $i$, and there is one consumer on platform $j$. Define $p_{i}^{i}$ as the price of the focal platform $i$ to consumers on platform $i$, and $\tilde{p}_{i}^{i}$ as the price of the focal platform $i$ to the consumer on platform $j$, if such a consumer exists. Likewise, define $p_{j}^{i}$ as the price of the nonfocal platform $j$ to consumers on the focal platform $i$, and $\tilde{p}_{j}^{i}$ as the price of the nonfocal platform $j$ to the consumer on platform $j$, if such a consumer exists. Notice that $p_{j}^{i}$ has the same interpretation as in our base model, while $\tilde{p}_{j}^{i}$ is the out-of-equilibrium price in the case where only one consumer switched. ${ }^{3}$

In what follows, we say that a Markov equilibrium is consistent with focality if at any date (a) the platform that wins the market becomes focal next period, and (b) the nonfocal platform cannot win the market if holding constant the Markov strategies of the platforms, there exists another outcome of the (dynamic) subgame where consumers (strictly) prefer to buy from the focal platform. Rephrasing, it means that consumers may buy from the nonfocal platform in equilibrium only if there is no other outcome where consumers prefer to buy from the focal platform. And thus, a nonfocal platform, in order to be active in the market needs to price in such a way that it eliminates all alternative outcomes in which consumers prefer to buy from the focal platform. This is a relatively strong notion of focality which aims at showing that even with patient firms, inefficiencies may prevail in the long-run due to network

\footnotetext{
${ }^{3}$ As one consumer is of mass 0 , we assume that other consumers are not affected when a single consumer deviates from equilibrium path, but for consistency of prices we assume that each platform optimizes the prices set for this deviating consumer (as it would be the case with a finite but large set of consumers).
} 
externalities and incumbency advantage.

We are interested in establishing the existence of an equilibrium where platform $i$ wins the market in all periods irrespective of whether it is focal or not, and would find it optimal to win back a consumer in the event the consumer had switched to the competing platform.

Consider an equilibrium in which platform $A$ wins when it is focal and when it is not. In this equilibrium, platform $B$ charges $p_{B}^{B}=p_{B}^{A}=0$ because as in our base model, platform $B$ cannot hold on to consumers at time $t+1$ even if it were to attract them at time $t$. As for platform $A$, it needs to set prices that satisfy

$$
q_{A}-p_{A}^{A}+\beta+\delta U^{A} \geq q_{B}-p_{B}^{A}-s+\delta\left(q_{A}-\tilde{p}_{A}^{A}-s+\beta+\delta U^{A}\right)
$$

where

$$
U^{i}=\frac{q_{i}-p_{i}^{i}+\beta}{1-\delta}
$$

The left-hand side is the consumer's utility from staying in platform $A$, given that all other consumers stay with $A$. If a consumer switches to $B$, the consumer expects to be alone in the current period. Then, in the next period, the focal platform $A$ charges this consumer $\tilde{p}_{A}^{A}$ which convinces this consumer to switch back to $A$. Then, once back on $A$, the consumer stays with $A$ in all future periods.

To ensure that these expectations are rational, a focal platform $A$ should be able to attract the deviating consumer back from the nonfocal platform $B$. That is:

$$
q_{A}-\tilde{p}_{A}^{A}+\beta-s+\delta U^{A} \geq q_{B}-\tilde{p}_{B}^{A}+\delta\left(q_{A}-\tilde{p}_{A}^{A}-s+\beta+\delta U^{A}\right),
$$

and $\tilde{p}_{B}^{A}=0$. That is, if a consumer switched in period $t-1$ from a focal platform $A$ to $B$, while $A$ remains focal at the beginning of period $t$, platform $B$ cannot hold on to this consumer; even at $\tilde{p}_{B}^{A}=0$, the consumer prefers to switch back to the focal platform $A$, over waiting another period and only then switch. Notice that platform $A$ 's profit from attracting back this marginal consumer is negligible, because of our assumption of price discrimination between existing and switching consumers and because consumers have continuum mass. Yet, to ensure that beliefs are consistent, the equilibrium requires that this marginal change in platform $A$ 's profit should be positive.

Suppose now that platform $A$ is nonfocal. A consumer joins the nonfocal platform $A$ if it is worthwhile to do so given the beliefs that all other consumers stay with platform $B$ in the current period, and that this consumer will switch back in the next 
period to the focal platform $B$ :

$$
q_{A}-p_{A}^{B}-s+\delta\left(q_{B}-\tilde{p}_{B}^{B}+\beta-s+\delta U^{B}\right) \geq q_{B}-p_{B}^{B}+\beta+\delta U^{B} .
$$

Notice that such a one-period deviation is the least beneficial deviation. Whatever benefit the consumer gets from being on $A$ is short-lived and she quickly needs to incur another switching cost. Note that if it is beneficial for a customer to deviate for one period, it will be even more beneficial to deviate and stay with $A$ for longer. That is, we demand from the nonfocal platform to be so attractive that users still want to incur switching costs even if they can benefit only for one period. We impose such a strong condition in the spirit of part (b) of the focality definition as stated above. If condition (9) holds there does not exist any equilibrium in which consumers want to stay with $B$ this period, even if they expect other consumers to stay with $B$.

Finally, consider the out-of-equilibrium outcome in which platform $B$ is focal at period $t$, and a consumer switched to $A$ at period $t-1$ and is alone in $A$. Notice that when conditions (1) to (3) hold, the deviating consumer knows that all customers of platform $B$ are switching to platform $A$ and will stay there forever. Hence despite $B$ being focal, this consumer stays with $A$ if it is worthwhile to do so given the beliefs that everybody else joins platform $A$ (this is in the spirit of divide and conquer strategies). We therefore need that

$$
q_{A}-\tilde{p}_{A}^{B}+\beta+\delta U^{A} \geq q_{B}-\tilde{p}_{B}^{B}-s+\delta\left(q_{A}-\tilde{p}_{A}^{A}+\beta-s+\delta U^{A}\right)
$$

and $\tilde{p}_{B}^{B}=0$.

Binding conditions (7) - (10) along with the 4 conditions $p_{B}^{B}=p_{B}^{A}=\tilde{p}_{B}^{B}=\tilde{p}_{B}^{A}=0$ define the 8 equilibrium prices. Solving, we have

$$
\begin{array}{cl}
p_{A}^{A}=\beta-\left(q_{B}-q_{A}\right)+s(1-\delta), & \tilde{p}_{A}^{A}=\beta-\left(q_{B}-q_{A}\right)-s(1+\delta), \\
p_{A}^{B}=-\beta-\left(q_{B}-q_{A}\right)-s(1+\delta), & \tilde{p}_{A}^{B}=\beta-\left(q_{B}-q_{A}\right)+s(1-\delta) .
\end{array}
$$

This equilibrium exists when the following conditions hold. First, a focal platform $A$ earns positive value from wining the market; that is, $A$ profitably wins the competition described by equation (7), i.e.,

$$
V_{A}^{A}(s) \equiv \frac{p_{A}^{A}}{1-\delta}=\frac{\beta-\left(q_{B}-q_{A}\right)}{1-\delta}+s \geq 0
$$


Second, a focal platform $A$ earns positive profit from attracting a consumer that moved to a nonfocal $B$ in the previous period, and then keeps this consumer in all future periods; that is, $A$ profitably wins the competition described by equation (8):

$$
\tilde{p}_{A}^{A}+\frac{\delta}{1-\delta} p_{A}^{A}=\frac{\beta-\left(q_{B}-q_{A}\right)}{1-\delta}-s \geq 0
$$

Third, a nonfocal platform $A$ earns positive profit from winning the market at period $t$, and then becoming focal in period $t+1$ onward; that is, $A$ profitably wins the competition described by equation (9):

$$
V_{A}^{B}(s) \equiv p_{A}^{B}+\delta V_{A}^{A}=\frac{\beta(2 \delta-1)-\left(q_{B}-q_{A}\right)}{1-\delta}-s \geq 0
$$

Fourth, a nonfocal $A$ earns positive profit from keeping a consumer that switched from $B$ to $A$ in the previous period, given that $A$ becomes focal in the next period onwards; that is, $A$ profitably wins the competition described by equation (10):

$$
\tilde{p}_{A}^{B}+\frac{\delta}{1-\delta} p_{A}^{A}=\frac{\beta-\left(q_{B}-q_{A}\right)}{1-\delta}+s \geq 0
$$

Notice that as $s \rightarrow 0$, then $V_{A}^{A}(s)$ and $V_{A}^{B}(s)$ converge to $V_{A}^{A}$ and $V_{A}^{B}$ in our base model without switching costs. Also, as in our base model, the binding condition from among (12), (13), (14) and (15) is $V_{A}^{B}(s) \geq 0$. Hence, such an equilibrium holds iff

$$
V_{A}^{B}(s) \geq 0 \quad \Longleftrightarrow \quad q_{B}-q_{A}<\beta(2 \delta-1)-s(1-\delta) .
$$

Recall that in our base model with only network effects, when $q_{B}>q_{A}$, this inefficient equilibrium exists iff $\delta>\frac{1}{2}$ and $q_{B}-q_{A}<\beta(2 \delta-1)$ (cf. Section 4 in our model). Yet, this equilibrium vanishes in a model with only switching costs (Section 5 in our model). Condition (16), which takes into account both network effects and switching costs, is a combination of these two polar cases. As $s \rightarrow 0$, condition (16) converges to $q_{B}-q_{A}<\beta(2 \delta-1)$. Moreover, as $s$ increases, this condition becomes tighter, implying that the region in which platform $A$ wins whether it's focal or not shrinks. This result is consistent with the findings of our paper - that the inefficient equilibrium emerges at high values of $\delta$ because of network effects and not switching costs. In a model with both $\beta>0$ and $s>0, \beta$ has a positive effect on condition (16) while $s$ has a negative effect.

Consider now an equilibrium in which platform $B$ wins whether it's focal or not. The analysis is symmetric to the analysis above, and we find that such an equilibrium 
exists iff

$$
V_{B}^{A}(s) \geq 0 \quad \Longleftrightarrow \quad q_{B}-q_{A}>\beta(1-2 \delta)+s(1-\delta) .
$$

Recall that in our base model with only network effects (Section 4), this equilibrium holds when $q_{B}-q_{A}>\beta(1-2 \delta)$. When the model only has switching costs (Section $5)$, this equilibrium holds when $q_{B}-q_{A}>s(1-\delta)$. Condition (17) is a combination of these two conditions. Intuitively, switching costs make it more difficult for a highquality but a nonfocal platform to win the market. This result is again consistent with the findings of our base model.

\section{Stochastic Qualities with a Uniform Distribu- tion: Welfare and Consumer Surplus}

This appendix extends Section 6 . We study how $\delta$ affects welfare and consumers' surplus. The following corollary shows that when the stochastic qualities are distributed according to a uniform distribution, average per-period social welfare is lower when $\delta=1$ than when $\delta=0$. In contrast, consumer surplus is increasing with $\delta$ at least when $\mu$ is sufficiently close to 0 .

Let $\bar{S}^{i}(i=A, B)$ denote the expected consumer surplus when platform $i$ is focal in period $t$, and let $S^{i}=(1-\delta) \bar{S}^{i}$ denote the average per-period expected consumer surplus. Recalling the definitions of $W^{A}$ and $W^{B}$ in the paper, we obtain the following result:

Corollary 1 (Welfare and consumer surplus under uniform distribution) Let $Q$ be uniformly distributed along the interval $[\mu-\sigma, \mu+\sigma]$, and suppose that $\sigma>2 \beta$ and $0 \geq \mu<\sigma+2 \frac{\beta^{2}}{\sigma}-3 \beta$. Then $W^{A}>W^{B}$ for all $0<\delta<1$ and $S^{A}>S^{B}$ for all $0 \leq \delta<1$. Moreover, $\left.W^{A}\right|_{\delta=0}=\left.W^{B}\right|_{\delta=0}>\left.W^{A}\right|_{\delta=1}=\left.W^{B}\right|_{\delta=1}$. Yet, $S^{A}$ and $S^{B}$ are increasing with $\delta$ when $\mu \rightarrow 0$.

\section{Proof:}

Substituting $F(Q)=\frac{Q-\mu+\sigma}{2 \sigma}$ into equation (11) from the proof to Proposition 3 yields:

$$
\bar{Q}^{A}=\beta-\frac{2 \delta \mu \beta}{\sigma-2 \delta \beta} \quad \text { and } \quad \bar{Q}^{B}=-\beta-\frac{2 \delta \mu \beta}{\sigma-2 \delta \beta} .
$$

To ensure that $\bar{Q}^{B}>\mu-\sigma$, we need $\sigma$ to be large enough that $\sigma>2 \beta$ and $0 \geq \mu<$ 
$\sigma+2 \frac{\beta^{2}}{\sigma}-3 \beta$. The recursive expected social welfare functions are then

$$
\begin{gathered}
\bar{W}^{A}=\int_{\mu-\sigma}^{\beta-\frac{2 \delta \mu \beta}{\sigma-2 \delta \beta}}\left(\beta+\delta \bar{W}^{A}\right) \frac{1}{2 \sigma} d q+\int_{\beta-\frac{2 \delta \mu \beta}{\sigma-2 \delta \beta}}^{\mu+\sigma}\left(\beta+q+\delta \bar{W}^{B}\right) \frac{1}{2 \sigma} d q, \\
\bar{W}^{B}=\int_{-\beta-\frac{2 \delta \mu \beta}{\sigma-2 \delta \beta}}^{\mu+\sigma}\left(\beta+q+\delta \bar{W}^{B}\right) \frac{1}{2 \sigma} d q+\int_{\mu-\sigma}^{-\beta-\frac{2 \delta \mu \beta}{\sigma-2 \delta \beta}}\left(\beta+\delta \bar{W}^{A}\right) \frac{1}{2 \sigma} d q .
\end{gathered}
$$

Therefore,

$$
\begin{aligned}
W^{A} & =(1-\delta) \bar{W}^{A} \\
& =\frac{1}{4}\left(4 \beta-\frac{\beta^{2}}{\sigma}+\sigma+\frac{\left.\mu\left(4 \delta^{2} \beta^{2}(2 \beta-3 \sigma)-\sigma^{2}(\mu+2 \sigma)+\delta \beta \sigma(5 \mu-4 \beta+10 \sigma)\right)\right)}{(\delta \beta-\sigma)(\sigma-2 \delta \beta)^{2}}\right), \\
W^{B} & =(1-\delta) \bar{W}^{B} \\
& =\frac{1}{4}\left(4 \beta-\frac{\beta^{2}}{\sigma}+\sigma+2 \mu+\frac{\left(\mu\left(8(-1+\delta) \delta^{2} \beta^{3}+\delta \beta(5 \mu-4(-1+\delta) \beta) \sigma-\mu \sigma^{2}\right)\right)}{(\delta \beta-\sigma)(\sigma-2 \delta \beta)^{2}}\right) .
\end{aligned}
$$

The gap $W^{A}-W^{B}$ can now be written as

$$
W^{A}-W^{B}=\frac{2(1-\delta) \delta \mu \beta^{2}}{(\sigma-\delta \beta)(\sigma-2 \delta \beta)} .
$$

As $\sigma>2 \beta$ (by assumption), $W^{A}-W^{B}>0$ for all $0<\delta<1$, and $W^{A}-W^{B}=0$ for $\delta=0$ and $\delta=1$. Moreover,

$$
\left.W^{A}\right|_{\delta=0}-\left.W^{A}\right|_{\delta=1}=\frac{\mu^{2} \beta^{2}(2 \sigma-\beta)}{\sigma(\sigma-\beta)(\sigma-2 \beta)^{2}}>0
$$

where the inequality follows because, by assumption, $\sigma>2 \beta$ and $\mu>0$.

Turning to consumer surplus, we have

$$
\begin{gathered}
\bar{S}^{A}=\int_{-\infty}^{\beta-\frac{2 \delta \mu \beta}{\sigma-2 \delta \beta}}\left(\beta-p_{A}^{A}+\delta \bar{S}^{A}\right) \frac{1}{2 \sigma} d q+\int_{\beta-\frac{2 \delta \mu \beta}{\sigma-2 \delta \beta}}^{\infty}\left(\beta+q-p_{B}^{A}+\delta \bar{S}^{B}\right) \frac{1}{2 \sigma} d q, \\
\bar{S}^{B}=\int_{-\beta-\frac{2 \delta \mu \beta}{\sigma-2 \delta \beta}}^{\infty}\left(\beta+q-p_{B}^{B}+\delta \bar{S}^{B}\right) \frac{1}{2 \sigma} d q+\int_{-\infty}^{-\beta-\frac{2 \delta \mu \beta}{\sigma-2 \delta \beta}}\left(\beta-p_{A}^{B}+\delta \bar{S}^{A}\right) \frac{1}{2 \sigma} d q .
\end{gathered}
$$


Substituting the prices from Section 6,

$$
\begin{aligned}
S^{A} & =(1-\delta) \bar{S}^{A} \\
& =\frac{1}{4}\left(2 \mu+4 \beta(1+\delta)-\frac{4 \mu(\mu+\beta(1-\delta))}{\sigma-\delta \beta}-\frac{3 \beta^{2}}{\sigma}-\sigma-\frac{10 \delta \mu^{2} \beta}{(\sigma-2 \delta \beta)^{2}}+\frac{\mu(3 \mu+8(1-\delta) \beta)}{\sigma-2 \delta \beta}\right), \\
S^{B} & =(1-\delta) \bar{S}^{B} \\
& =\frac{1}{4}\left(2 \mu+4 \beta(1+\delta)-\frac{4 \mu(\mu-\beta(1-\delta))}{\sigma-\delta \beta}-\frac{3 \beta^{2}}{\sigma}-\sigma-\frac{10 \delta \mu^{2} \beta}{(\sigma-2 \delta \beta)^{2}}+\frac{\mu(3 \mu-8(1-\delta) \beta)}{\sigma-2 \delta \beta}\right) .
\end{aligned}
$$

We have

$$
S^{A}-S^{B}=\frac{2(1-\delta) \mu \beta \sigma}{(\sigma-\delta \beta)(\sigma-2 \delta \beta)} .
$$

Hence, $S^{A}>S^{B}$ for all $\delta<1$. Moreover,

$$
\left.\frac{\partial S^{A}}{\partial \delta}\right|_{\mu=0}=\left.\frac{\partial S^{B}}{\partial \delta}\right|_{\mu=0}=\beta>0 .
$$

Notice that $S^{A}$ and $S^{B}$ are continuous in $\mu$. Hence, they are increasing with $\delta$ as long as $\mu$ is not too high.

This completes the proof of Corollary 1. 